\title{
Improvement of Accuracy of Global Numerical Weather Prediction Using Refined Error Covariance Matrices
}

\author{
TOSHIYUKI ISHIBASHI \\ Meteorological Research Institute, Japan Meteorological Agency, Tsukuba, Japan
}

(Manuscript received 16 August 2019, in final form 9 April 2020)

\begin{abstract}
In data assimilation for NWP, accurate estimation of error covariance matrices (ECMs) and their use are essential to improve NWP accuracy. The objective of this study is to estimate ECMs of all observations and background variables using sampling statistics, and improve global NWP accuracy by using them. This study presents the first results of such all ECM refinement. ECM diagnostics combining multiple methods, and analysis and forecast cycle experiments were performed on the JMA global NWP system, where diagonal components of all ECMs and off-diagonal components of radiance observations are refined. The ECM diagnostic results are as follows: 1) the diagnosed error standard deviations (SDs) are generally much smaller than those of the JMA operational system (CNTL); 2) interchannel correlations in humidity-sensitive radiance errors are much larger than 0.2; and 3) horizontal correlation distances of AMSU-A are $\sim 50 \mathrm{~km}$, excluding channel 4. The experimental results include the following: 1) the diagnosed ECMs generally improve forecast accuracy over CNTL even without additional tunings; 2) the supplemental tuning parameter, which is the deflation factor (0.6 in SD) applied for the estimated ECMs of nonsatellite conventional data and GPS radio occultation data, statistically significantly improves forecast accuracy; 3 ) this value 0.6 is set as the same value as the ratio of the estimated background error SD to that in CNTL; 4) high-density assimilation (10 times) of AMSU-A is better than CNTL, not better than that with 5 times; and 5) ECMs estimated using boreal summer data can improve forecast accuracy in winter, which indicates their robustness.
\end{abstract}

\section{Introduction}

In data assimilation for numerical weather prediction (NWP), observation data are one of the most important sources of information. Millions of observation data are assimilated in each analysis by a data assimilation system (DAS) for NWP. However, observation data currently assimilated are a very small part of the observation data that is potentially available. Even when the observation data are limited to that which comes from the Global Telecommunication System (GTS), the assimilated data are a few percent only of the total. For example, Bauer et al. (2011) describe only $5 \%$ of satellite observations are actually assimilated in European Centre for Medium-Range Weather Forecasts (ECMWF). Note that the percentage of assimilated observation information is larger than this value because of observation error correlations. This limitation comes from various approximations in NWP systems. Insufficient approximation accuracy of observation error covariance

\footnotetext{
Corresponding author: T. Ishibashi, ishibasi@mri-jma.go.jp
}

matrices (OECMs) is one of the primary limitations. Other major limitations include insufficient forecast accuracy for observed quantities, strong nonlinearity of observation operators, and increase of computational cost. For example, when a DAS ignores horizontal observation error correlations, thinning of the observation data are necessary to satisfy the assumption that observation data do not have error correlation. If the correlation structure is not well known, the thinning distance employed in the DAS to safely exclude the observation error correlations would be much longer than the true correlation distance. When a DAS uses a diagonal OECM ignoring existing interchannel correlations, observation error variances must be artificially inflated. Therefore, accurate OECM estimation and its use in the DAS (refinement of OECMs) is essential for assimilating more observation information. However, the accurate estimation of error covariance matrix (ECM) is a difficult scientific problem because the true atmospheric state is not known. In data assimilation, observation errors include not only measurement errors but also include representativeness errors and errors of 
observation operator (Lorenc 1986; Kalnay 2003), and the latter two error components are not known as accurate as the first component. OECMs in current NWP systems have aspects as tuning parameters determined by trial and error for each dataset to maintain the accuracy of NWP. Furthermore, it is necessary to simultaneously refine a background ECM (BECM).

For OECM estimation, there are five primary diagnostic methods, including the method developed by Hollingsworth and Lönnberg (1986, hereafter HL method), the method of Desroziers et al. (2005, hereafter D05 method), the method of Desroziers and Ivanov (2001), the method using randomization (Andersson et al. 2000, hereafter randomization method), and the method of Daescu (2008). However, all of these estimation methods are based on some assumptions, and there is no perfect method. The HL method can have large estimation errors due to the observation error correlation, the D05 method cannot completely separate observation errors and background errors, and the randomization method can have large estimation errors when a BECM used in a DAS is significantly different from true one (Bormann and Bauer 2010). Therefore, comparisons of ECMs estimated by different methods, and evaluation of them with analysis and forecast cycle experiments (hereafter, cycle experiment) are still needed.

In recent years, studies have clarified that OECMs estimated by the different methods qualitatively agree with each other for some sensors of radiance observations. Bormann and Bauer (2010) and Bormann et al. (2010) showed such agreement with three methods: the D05 method, the HL method, and the randomization method for radiance data from microwave sounders [the Advanced Microwave Sounding Unit-A (AMSU-A), Microwave Humidity Sounder (MHS)] and infrared sounders [the High Resolution Infrared Radiation Sounder (HIRS), the high-spectral-resolution Atmospheric Infrared Sounder (AIRS), the Infrared Atmospheric Sounding Interferometer (IASI)]. Stewart et al. (2014) also estimated OECMs of IASI using the D05 method. Bormann et al. (2013) and Campbell et al. (2017) estimated OECMs of Advanced Technology Microwave Sounders (ATMS) using the D05 method. Furthermore, the use of estimated OECMs in DASs for NWP has been started. Interchannel observation error correlation of data from infrared sounders [AIRS, IASI, and the Cross-track Infrared Sounder (CrIS)] are operationally used with readjusted eigenvalues or variances (Weston et al. 2014; Bormann et al. 2016; Eresmaa et al. 2017). These studies were performed on the model space four-dimensional variational (4DVar) DASs at ECMWF and the Met Office. Campbell et al. (2017) showed forecast accuracy improvement by introducing diagnosed OECMs for
ATMS and IASI in their observation space 4DVar DAS, which is known as the Naval Research Laboratory (NRL) Atmospheric Variational Data Assimilation System Accelerated Representer (NAVDAS-AR). These studies show the OECM diagnostics have enough accuracy to improve current NWP for these observations.

However, these previous studies targeted satellite radiance data, and implementation of diagnosed OECMs has been limited to just a few types of sensors, such as ATMS and hyper spectral sounders [(HSS) AIRS, IASI, CrIS]. Therefore, studies including other observation datasets, such as humidity-sensitive microwave radiance observations, conventional observations such as radiosondes, and GPS radio occultations (GPSRO) are needed, and a BECM must also be refined. The robustness of the estimated OECMs must also be evaluated for use in NWP. Furthermore, the origin of the inflation factor applied to estimated OECMs used in the previous studies remains unclear.

The objective of this study is to estimate OECMs of all observation datasets and a BECM using sampling statistics, and use them to improve the accuracy of global NWP. To accomplish this, ECM diagnostics combining multiple ECM estimation methods, and analysis and forecast cycle experiments are performed on the global NWP system at Japan Meteorological Agency (JMA). Interchannel correlations of satellite radiance data, error variances of all data (radiances, conventional observations, GPSRO, and background field variables) are estimated and used. On the other hand, nondiagonal components of the BECM and OECMs other than radiance observations are not changed. Thinning distances of AMSU-A data are also refined based on the estimated OECM. The D05 method is used primarily for these ECM estimations combined with the randomization and HL methods.

The remainder of this paper is organized in the following manner. In section 2, the three different ECM estimation methods are briefly reviewed and the implementation methods of the estimated ECMs are presented. In section 3, the experimental design of the ECM estimation and cycle experiments are described. In section 4, the structure of the estimated ECMs and their impact on NWP are evaluated using cycle experiments. Finally, a summary and conclusions is presented in section 5. Appendix A describes the consideration of the estimated BECM. Appendix B describes results of some extra experiments.

\section{Methods}

\section{a. Brief review of the three ECM estimation methods}

Here, the three ECM estimation methods, the D05 method, the HL method, and the randomization method, 
which are used in this study, are briefly reviewed. The HL method and the D05 method estimate ECMs from observation minus background field in observation space $(\mathrm{OmB})$ statistics, and observation minus analysis field in observation space $(\mathrm{OmA})$ statistics, based on different assumptions. The HL method works well when horizontal error correlations of observation errors are negligible. The D05 method works well when the correlation distances and variances for observation errors and those for errors of background counterparts are significantly different (D05). While some limitations of this method have been identified (Ménard 2016; Waller et al. 2016), the validity of OECMs estimated by this method has been demonstrated in real NWP systems (section 1). The randomization method is used to estimate background error statistics in the observation space in this study. In the following description, "observation data" refers to "bias-corrected observation data" by bias-correction schemes such as the variational bias-correction scheme (VarBC; Derber and Wu 1998; Dee 2004).

\section{1) THE D05 METHOD}

The D05 method diagnoses OECM, R, as follows:

$$
R_{p, q}=\frac{1}{M} \sum_{m}\left(o_{p}^{m}-b_{p}^{m}\right)\left(o_{q}^{m}-a_{q}^{m}\right),
$$

where indices $p$ and $q$ are the integrated indices summarizing all the information needed to identify each observation, such as observation location, time, and channel, run from one to the total number of observations $P, m$ denotes the sampling ensemble members that runs from one to the total sample size $M, R_{p, q}$ is the $(p, q)$ th component of $\mathbf{R}, o_{p}^{m}, b_{p}^{m}$, and $a_{p}^{m}$ denote the $p$ th components of the observation vector $\mathbf{o}$, the background state vector in observation space $\mathbf{b}$, and the analysis state vector in observation space a, for the $m$ th sample, respectively. The ensemble average in Eq. (1) can be replaced by the space-time average in real calculations, assuming that the error statistics are uniform in space and time. The D05 method diagnoses a BECM in observation space $\mathbf{G}$ as follows:

$$
G_{p, q}=\frac{1}{M} \sum_{m}\left(o_{p}^{m}-b_{p}^{m}\right)\left(a_{q}^{m}-b_{q}^{m}\right) .
$$

In this study, the ratio of the estimated error standard deviation (SD) to that of the original SD is referred to as "SD ratio (SDR)."

Here, we need to consider the validity of this method when used in NWP DASs. This is because whereas Eq. (1) is true only when the ECMs used in a DAS are true, this is not the case in the NWP DASs. In fact, it has been shown mathematically that true ECMs are never obtained by this method in a suboptimal DAS where ECMs and a Kalman gain are incorrect (Ménard 2016). On the other hand, what is important in the ECM estimation for real DASs is whether ECMs closer to the true ones than original ECMs can be obtained. Qualitative validity of the D05 method in this sense can be considered by rewriting Eq. (1) in the filtering form as shown in D05. That is $\tilde{\mathbf{X}}^{\prime}=\tilde{\mathbf{X}}\left(\tilde{\mathbf{D}}^{-1} \mathbf{D}\right)$, where $\tilde{\mathbf{X}}$ and $\tilde{\mathbf{X}}^{\prime}$ are the ECM (OECM or BECM in the observation space) used in the DAS and estimated by the D05 method, respectively, $\tilde{\mathbf{D}}$ and $\mathbf{D}$ are the OmB covariance matrix used in the DAS and the true one, respectively. This shows the D05 method estimates the ECM by multiplying the original ECM by the coefficient matrix $\tilde{\mathbf{D}}^{-1} \mathbf{D}$ representing the mismatch of system's and true OmB covariance matrices. Therefore, the method basically works to correct suboptimality of ECMs, where the coefficient matrix is not exactly correct one for each ECM, but averaged one for the OmB covariance matrices. In fact, in homogeneous ideal cases, D05 shows when the correlation distances for observation errors and those for errors of background counterparts are significantly different the method can improve ECMs. More recently, Waller et al. (2016) shows even when the correlation distances for observation errors and those for errors of background counterparts are similar the method can improve ECMs in some cases. The validity of OECMs estimated by this method has been demonstrated for some sensors in real NWP systems (section 1). These considerations show that the D05 method can generate approximately valid ECMs even in a real NWP system for some cases. On the other hand, the filtering form Eq. (1) also shows the explicit limitation of the D05 method as mentioned above. That is the coefficient matrix $\tilde{\mathbf{D}}^{-1} \mathbf{D}$. For example, when $\tilde{\mathbf{D}}$ is correct, the D05 method cannot correct ECMs used in the DAS even when they are incorrect. Whether ECMs estimated by the D05 method are correct can be verified by comparisons of ECMs estimated by different methods, and evaluation of them with cycle experiments.

\section{2) THE HL METHOD}

In this study, the HL method is used to estimate the interchannel correlations to compare them with those estimated by the D05 method. We assume observations that are horizontally separated by more than distance $d_{1}$ have no correlation, and changes of background covariance values in the observation space within distance $d_{2}$ are negligibly small. This indicates that when observations are horizontally thinned with a distance longer than $d_{1}, \mathbf{R}$ is a block diagonal for each horizontal observation point, and in each block, interchannel correlations 
are considered as off-diagonal components of $\mathbf{R}$. Such a block can be estimated as follows:

$$
R_{p, q}^{\mathrm{HL}}=D_{p, q}-G_{p, q}^{\mathrm{HL}} .
$$

Here, indices $p$ and $q$ denote channels of satellite radiance observations, $D_{p, q}=(1 / M) \sum_{m}\left(o_{p}^{m}-b_{p}^{m}\right)\left(o_{q}^{m}-b_{q}^{m}\right)$ is the $(p, q)$ th component of the departure (OmB) covariance matrix, $G_{p, q}^{\mathrm{HL}}=(1 / N) \sum_{d_{1}<d(r, s)<d_{2}} D_{r, s}$ is the $(p, q)$ th component of the BECM in the observation space diagnosed by the HL method, where the summation is taken for observation pairs $(r, s)$ whose distances $d(r, s)$ satisfy $d_{1}<d(r, s)<d_{2}$, and $N$ is the number of such data pairs.

\section{3) THE RANDOMIZED METHOD}

The randomization method generates realizations of the background error statistics described by the BECM in observation space used in the DAS, which denoted by $\mathbf{G}^{\mathrm{RD}}$, can then be estimated as

$$
\mathbf{G}^{\mathrm{RD}}=\frac{1}{M} \sum_{m}\left(\mathbf{H B}^{1 / 2} \mathbf{s}^{m}\right)\left(\mathbf{H B}^{1 / 2} \mathbf{s}^{m}\right)^{\mathrm{T}},
$$

where $\mathbf{B}^{1 / 2}$ is the square root of the BECM used in the DAS, $\mathbf{s}^{m}$ is the $m$ th sample of the random number vector obeying standard normal distribution, and $\mathbf{H}$ is the tangent linear observation operator. Therefore, we can estimate the SDR of the BECM in the observation space by comparing Eq. (2) with Eq. (4):

$$
\mathrm{SDR}=\sqrt{\sum_{q \in A} G_{q, q} / \sum_{p \in A} G_{p, p}^{\mathrm{RD}},}
$$

where $A$ denotes the arbitrary part of G's diagonal elements, for example, temperature observation data at $500 \mathrm{hPa}$ from radiosondes. Although, the OECM also can be estimated, assuming the implemented $\mathbf{B}$ is correct, this assumption is not sufficiently satisfied in real DASs. Indeed, Bormann and Bauer (2010) reported that in some cases B estimated with this method conflicted with $\mathrm{OmB}$ statistics. Therefore, the randomization method is used only for the SDR estimation Eq. (5) in this study.

\section{b. Implementation of estimated ECMs into a 4DVar DAS}

\section{1) INTERCHANNEL CORRELATIONS}

To implement the estimated nondiagonal OECMs of radiance observations including interchannel correlations on a 4DVar DAS, the direct inverse calculation approach can be used, which calculates the inverse of OECMs for each observation profile and
4DVar iteration. This approach has been used in previous studies (Weston et al. 2014; Bormann et al. 2016; Eresmaa et al. 2017) to introduce interchannel correlations of HSS and ATMS. The inverse calculations for every horizontal observation points and 4DVar iterations are necessary to treat different channel selection for each profile and to avoid large computer memory use. The computational cost of these OECM inverse calculations is not dominant in 4DVar, where the primary cost is associated with forecast model integrations, due to the small degrees of freedom of each OECM and computationally efficient algorithms based on the Cholesky decomposition (Golub and Van Loan 1996). Therefore, this method is conceptually simple and computationally possible.

\section{2) SuPPLEMENTAL TUNING PARAMETER FOR ECMS ESTIMATED BY THE D05 METHOD}

As noted in sections 1 and 2, all the existing ECM estimation methods have their assumptions, and limitations because of those assumptions. Therefore, it is reasonable to expect that some supplemental tunings for the estimated ECMs to account for such limitations have to be included when we use them in real NWP systems. Furthermore, if the estimation of ECMs is done only for specific datasets, then some supplemental tunings are also needed. For example, when only OECMs are estimated by the D05 method without estimation of BECMs, such supplemental tuning is needed as a proxy of the BECM estimation. In this study, the supplemental tuning is done by deflating the variances of the estimated ECMs for selected observation types. The magnitude of the deflation factor for such supplemental tuning can be limited by the SDRs in any cases. Since the supplemental tuning is an ad hoc tuning, it should be as simple as possible. As will be shown in section 4 , in this study, we use only one supplemental tuning parameter for all selected observation types (nonsatellite conventional observations and GPSRO) with the same value of the SDR for the BECM.

\section{Experimental design of ECM estimation and cycle experiments}

Estimations of ECMs using the methods described in the previous section and cycle experiments to evaluate their impacts on analysis and forecast accuracy are designed as follows. These experiments were conducted on the JMA global NWP system (JMA 2013), which is the version that was in operation until 
TABLE 1. Observation datasets assimilated in the JMA global DAS. AMSU-A: Advanced Microwave Sounding Unit-A. MHS: Microwave Humidity Sounder. CSR: clear sky radiance. SSMIS: Special Sensor Microwave Imager/Sounder. GNSS: Global Navigation Satellite System. SAPHIR: Sondeur Atmosphérique du Profil d'Humidité Intertropicale par Radiométrie.

\begin{tabular}{ll}
\hline \hline Dataset name & Description \\
\hline AMSU-A & Radiance data of AMSU-A instruments from MetOp-1/2,NOAA-15/18/19, and Aqua \\
MHS & Radiance data of MHS instruments from MetOp-1/2,NOAA-18/19 \\
MWI & Radiance data of microwave imager instruments: GMI from GPM Core Observatory, and AMSR2 from GCOM-W1 \\
SAPHIR & Radiance data of SAPHIR instrument from Megha-Tropiques \\
CSR & Radiance data of geostationary satelliete water vapor channels from Himawari-8, GOES-13/15, and Meteosat-7/10 \\
SSMIS & Radiance data of SSMIS instruments from DMSP17, 18 \\
HSS & Radiance data of hyper spectral sounder instruments AIRS and IASI from Aqua and MetOp-1/2, respectively \\
GPSRO & Global positioning system radio occultation (GPSRO) data in bending angle from $M e t O p-1 / 2$, COSMIC, and \\
& TerraSAR-X \\
SURF & Land surface reports of pressure \\
SONDE & Radiosonde obserbations (temperature, wind, relative humidity) and pilot balloon observations (wind) \\
AVIATION & Upper-air observations by aircrafts (temperature and wind) \\
BOGUS & Pseudo-observation data in western pacific generated for tropical cyclone structure \\
WPR & Wind profiler data in Japan, Hong Kong, Europe, and the United States \\
AMV-GEO & Atmospheric motion vector (AMV) data retrieved from images of geostationary satellites (GEOs) \\
AMV-POL & AMV data retrieved from images of polar orbitting satellites (POs), and from combinatin of PO and GEO images \\
SCAT & Ocean surface wind data from ASCAT scatterometer instruments from $M e t O p-1 / 2$ \\
GNSS_SURF & Atmospheric signal delay measurements of the ground-based GNSS receivers \\
\hline
\end{tabular}

May 2017. The horizontal resolution of the global NWP model (outer model) is about $20 \mathrm{~km}$, the number of vertical layers is 100 , and the top level of the model is $0.01 \mathrm{hPa}$. The horizontal resolution of the inner models (adjoint model and tangent linear model) is about $60 \mathrm{~km}$ with the same vertical levels as those of the outer model. The DAS is an incremental 4DVar with a 6-h data assimilation window and one outer loop. The VarBC scheme is used for bias correction of satellite radiance data. Table 1 shows the assimilated observation data. Basic properties of observation impacts in the JMA NWP system have been presented by Ishibashi (2018). In addition to the dataset names listed in Table 1, several integrated dataset names are also used, as follows. TBB refers to the entire set of radiance observations, $\mathrm{CNV}$ refers to the entire set of conventional observations (observations other than TBB and GPSRO). The OECMs used in the operational system is diagonal. They have been set based on $\mathrm{OmB}$ statistics, and partly based on the D05 method only for SONDE (except for humidity observations), AVIATION, SURF, and AMSU-A data with inflation parameters for each dataset, channel, and element determined by trial and error using an older and lower-resolution version NWP system (JMA 2007; Kadowaki and Yoshimoto 2012). The BECM used in the JMA operational system has been estimated based on the NMC method (Parrish and Derber 1992). The rescaling factor used in the NMC method, which converts 24 -h forecast error differences into 6-h forecast errors, is set to 0.9 for SDs in the JMA system (JMA 2013).
The new ECM estimation with the methods described in section 2 was conducted using 1-month observation data in August 2016 for diagonal components of OECMs for CNV and GPSRO, and those of the BECM. Since, in this study, OECMs are estimated based on the assumption of global uniformity, and estimated only for their diagonal components for CNV and GPSRO, we can consider the 1-month observation data are sufficient. For TBB, 5 days (20 analyses) of data (from 0000 UTC 20 August to 1800 UTC 24 August 2016) were used for the OECM estimation including interchannel correlation. Since the amount of satellite radiance data are much larger than other observation data, the sampling number is still larger than that of 1-month CNV data. Weston et al. (2014) has shown that only 1 day of data is enough for estimate interchannel correlation for IASI. In all these ESM estimations, bias-corrected observation data in the operational system were used. For example, the global average of $\mathrm{OmB}(\mathrm{OmA})$ for AMSU-A data is smaller than $3 \%$ of the SD of OmB $(\mathrm{OmA})$, and biases are sufficiently small in this dataset. In the estimates of horizontal observation error correlations, observations are separated into $25 \mathrm{~km}$ bins. This estimation is executed using results of a cycle experiment with $25 \mathrm{~km}$ thinning distances for radiance observations.

The threshold distances $d_{1}$ and $d_{2}$ used in the estimation of channel correlation by the HL method described in section $2 \mathrm{a}(2)$ are 100 and $200 \mathrm{~km}$, respectively. By the assumption about $d_{2}$ [section $2 \mathrm{a}(2)$ ], the estimated BECM using $d_{1}<d<d_{2}$ data is used as the 
TABLE 2. List of experiments.

\begin{tabular}{|c|c|c|c|c|c|}
\hline Experiment name & Description & New ECM & Supplemental tuning & AMSU-A density & Season \\
\hline CNTL & JMA original NWP system & NO & NO & 1 & Summer \\
\hline EXP-BR-A5 & $\begin{array}{l}\text { Using the refined } \mathbf{R} \text { and } \mathbf{B} \text { based on the } \\
\text { D05 estimation, and } 5 \text { times higher } \\
\text { density assimilation of AMSU-A }\end{array}$ & YES & NO & 5 & Summer \\
\hline EXP-BRT-A5 & $\begin{array}{l}\text { Same as EXP-BR-A5 except for using the } \\
\text { supplemental tuning parameter }\end{array}$ & YES & YES & 5 & Summer \\
\hline EXP-BRT-A10 & $\begin{array}{l}\text { Same as EXP-BRT-A5 except for assim- } \\
\text { ilationg AMSU-A with } 10 \text { times higher } \\
\text { density }\end{array}$ & YES & YES & 10 & Summer \\
\hline EXP-BRT-A1 & $\begin{array}{l}\text { Same as EXP-BRT-A5 except for no high } \\
\text { density assimilation of AMSU-A. }\end{array}$ & YES & YES & 1 & Summer \\
\hline EXP-BR-A1 & $\begin{array}{l}\text { Same as EXP-BR-A5 except for no high- } \\
\text { density assimilation of AMSU-A }\end{array}$ & YES & NO & 1 & Summer \\
\hline EXP-BRT-A5-WIN & $\begin{array}{l}\text { Same as EXP-BRT-A5 except for winter } \\
\text { experiment }\end{array}$ & YES & YES & 5 & Winter \\
\hline CNTL-WIN & JMA original NWP system & NO & NO & 1 & Winter \\
\hline
\end{tabular}

BECM for $d<d_{1}$ without extrapolation for Eq. (3). This no-extrapolation approach has been used previous studies such as Bormann and Bauer (2010). Whereas this approach is free from the dependence of the estimation results on the selected extrapolation function form, it is potentially affected by changes of background error correlation within the distance $d<d_{1}$, which tends to result in overestimation of OECMs. In contrast, if OECM for the distance $d>d_{1}$ has error correlations, then OECM tend to be underestimation. The setting values used here, $d_{1}=100 \mathrm{~km}$ and $d_{2}=200 \mathrm{~km}$ are selected to satisfy the assumptions for them [section $2 \mathrm{a}(2)$ ], as follows. First, the $d_{1}$ value is set as the distance where observation error correlations for the distance $d>d_{1}$ are negligible for most radiance observations. As show in the next section, this is approximately satisfied. Second, the $d_{2}$ value is set as the distance where changes of BECM within the distance are small enough. For example, the BECM changes for AMSU-A within the distance $200 \mathrm{~km}$ is about $10 \%$ to $20 \%$ for all channels. Therefore, we can expect OECMs estimated by the HL method using these settings are approximately valid. Finally, since there was no deterioration of 4DVar convergence, the diagnosed correlation matrices were not adjusted to make the condition number close to 1 .

The 1-month analysis and forecast cycle experiments for boreal summer and winter terms were constructed (Table 2). The summer experiments were conducted for the period from 0000 UTC 20 July to 1800 UTC 31 August 2016, and the winter experiments were conducted for the period from 0000 UTC 20 November to 1800 UTC 31 December 2016. Since the first 12 days are the spinup period, they are not used for the diagnostics of ECMs or the initial conditions of forecasts to be verified. The evaluation period for the summer experiments is August 2016, and that for the winter experiments is December 2016. Control experiments with the JMA operational NWP system were executed both in summer (CNTL) and winter (CNTL-WIN) as reference experiments for comparison with test experiments. We performed the following two main test experiments to clarify effects of the refined ECMs in NWP. The first experiment (named EXP-BR-A5) is an experiment using the estimated OECMs for all observations and an estimated BECM by the D05 method, where the nondiagonal OECMs are used for the TBB, while other OECMs and the BECM are refined only for variances. The nondiagonal OECMs for the TBB are introduced by the direct matrix inverse calculation approach (Weston et al. 2014; Bormann et al. 2016; Eresmaa et al. 2017) as described in section $2 b(1)$. The thinning distance for the AMSU-A data is also refined according to the estimated horizontal correlation structure, as follows. First, a maximum correlation distance (MCD) at which the observation error correlations become smaller than 0.2 is determined from the estimated ECMs, where the value 0.2 is used as the largest negligible correlation according to previous studies such as Liu and Rabier (2003). Second, we determine a new thinning distance of AMSU-A data as a distance not less than the MCD (section 4a). The thinning distance is set $110 \mathrm{~km}$ in EXP-BR-A5 against $250 \mathrm{~km}$ in CNTL. This value is set as a minimum distance larger than MCDs of all AMSU-A channels. Consequently, the assimilation density of the AMSU-A data is approximately 5 times greater than that of CNTL. This test was executed to clarify the effect of using the estimated ECMs by the D05 method including the thinning distance refinement. The second experiment (referred to as EXP-BRT-A5) is 
an experiment employing the supplemental tuning [section $2 \mathrm{~b}(2)$ ], where a deflation factor (0.6) in observation error SDs for non-satellite-based observations in CNV (nonsatellite CNV) and GPSRO is introduced as the supplemental tuning parameter. The value of this deflation factor is determined by setting it at the same value of the SDR for the BECM [section 4(a)], and the nonsatellite CNV and GPSRO are selected due to their anchor effects for the VarBC (Auligné et al. 2007) and the results of EXP-BR-A5 experiment shown in section 4. The thinning distance for AMSU-A is the same as that used for EXP-BR-A5. This experiment was executed to clarify the effect of the supplemental tuning for the estimated ECMs.

Four additional experiments are also performed, as follows. The first additional experiment (referred to as EXP-BRT-A10) is the same as EXP-BRT-A5 except that the assimilation density of the AMSU-A data is approximately 10 times greater than that of CNTL. The thinning distance of AMSU-A is about $80 \mathrm{~km}$, which is $30 \mathrm{~km}$ shorter than the thinning distance used in EXPBRT-A5. This value $30 \mathrm{~km}$ is set as a value nearly corresponding to the distance of 1 bin used in the MCD estimation and the distance of the model grid. This experiment was executed to evaluate the effects of such a high-density AMSU-A assimilation, the effects of the perturbation of the MCD, on NWP accuracy. The second additional experiment (referred to as EXP-BRTA5-WIN) is the winter experiment whose settings are the same as EXP-BRT-A5, where the same ECMs as the summer experiment are used. This experiment was executed to check the robustness of estimated ECMs. The third additional experiment (referred to as EXP-BRA1) and the fourth additional experiment (referred to as EXP-BRT-A1) are the same as EXP-BR-A5 and EXP-BRT-A5, respectively, except that the assimilation density of the AMSU-A data is the same as that of CNTL. Although, these two refinements (ECM refinement and thinning distance refinement) basically should be executed simultaneously, since thinning distance is determined from the MCD described in the refined ECM, these experiments were executed to separate effects of the ECM refinement and the high-density AMSU-A assimilation. (In appendix B, the inverse cases are described, where only thinning distances of AMSU-A data are changed without the ECM refinement.)

Forecast error is measured using ERA5 (Hersbach and Dee 2016) as truth. Other verification methods using $\mathrm{CNV}$ as truth and $\mathrm{OmB}$ statistics are also employed. Because of its clear advantages in coverage, average accuracy, and high independency to JMA forecasts, we use the ERA5 verification as the main verification metric. It should be noted that since any verification method assumes forecast error differences between two experiments are larger than reference analysis or observation errors, the verification of forecasts close to their initial times, or for variables or areas with small error growth rate are difficult. In addition, it should be noted that the bias correction for temperature and height of SONDE and temperature of AVIATION data are routinely executed based on OmB statistics from a previous month in the JMA operational system, and these data are used as truth in the verification using CNV. Statistical significance is evaluated with the $t$ test of paired samples for mean differences under serial dependence (Wilks 2011).

\section{Results}

\section{a. Estimated ECMs}

Figure 1 shows diagnosed observation error SDs for all observation datasets by the D05 method. The estimated error SDs are generally much smaller than those use in CNTL. More specifically, the error SDs for parts of TBB, GPSRO, and the satellite-based observations in CNV (satellite-based CNV) are smaller than half of those used in CNTL. The observation dataset with the largest inflation in CNTL is the water vapor sensitive microwave radiances (MWI, MHS, SAPHIR, and SSMIS). These results agree with diagnostics using different observation error estimation methods, the method of Desroziers and Ivanov (2001) and the method of Daescu (2008), reported in Ishibashi (2010). Introduction of the estimated OECM could bring much more observation information into the JMA DAS.

Figure 2 shows the diagnosed horizontal observation error correlations for AMSU-A as a function of horizontal distances derived by the D05 method. This figure shows the MCDs are about $50 \mathrm{~km}$ except for channel 4 where the MCD is about $100 \mathrm{~km}$. These MCDs are much shorter than the current thinning distance used in the JMA DAS, which is $250 \mathrm{~km}$, and that of other NWP centers, for example, $1.25^{\circ}$ (about $135 \mathrm{~km}$ ) in ECMWF (Bauer et al. 2011). Therefore, higher density assimilation of AMSU-A data would be possible and improve the accuracy of the analysis. These short MCDs for AMSU-A agree with the results of Bormann and Bauer (2010). MCDs for the water vapor sensitivity sensors (such as MHS, SAPHIR) are about 100 to $150 \mathrm{~km}$ (not shown). Finally, the horizontal functional form differences between OECMs and BECMs in the observation space shown in Figs. 2e and $2 \mathrm{f}$ support the validity of the estimation by the D05 method [section 2(a)]. 


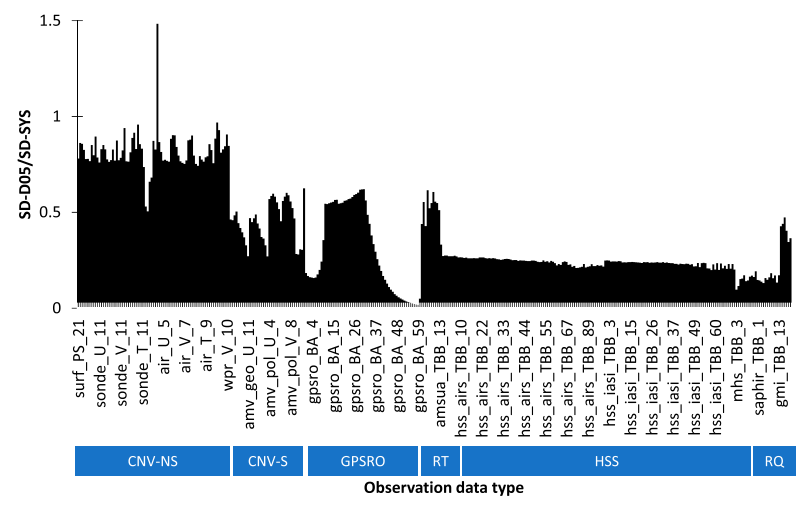

FIG. 1. Observation error SDs estimated by the D05 method. The vertical axis is the ratio of observation error SDs estimated by the D05 method (SD-D05) to those used in the JMA operational system (SD-SYS) (SDR). The horizontal axis shows observation datasets, where dataset names are shown at 11-point intervals formatted in $A A \_B B \_C C$. $A A$ denotes dataset names (see Table 1). AVIATION is abbreviated here as "air." $B B$ denotes assimilating elements, zonal wind $(U)$, meridional wind $(V)$, temperature $(T)$, relative humidity $(\mathrm{RH})$, bending angle (BA), and blackbody brightness temperature (TBB). $C C$ denotes pressure levels ( $\times 100 \mathrm{hPa}$, except for " 21 ," which denotes surface) for conventional observations, heights $(\mathrm{km})$ for BA, and channels for TBB. The integrated observation dataset names are also shown in the box below the horizontal axis, where CNV-NS and CNV-S denote the non-satellite-based and satellite-based observations in $\mathrm{CNV}$, respectively, $\mathrm{RT}$ and $\mathrm{RQ}$ denote the temperature and humidity-sensitive sensors in TBB other than HSS, respectively.

Figures $3 a-f$ show the interchannel correlations diagnosed by the D05 method for some selected radiance observations. The interchannel correlations for humidity-sensitive sensors, MHS, AMSR2, AHI, and SAPHIR are larger than 0.2. Therefore, these interchannel correlation must be treated in DASs. In contrast, interchannel correlations for temperature sensitive sensors, AMSU-A, and upper-level observing IASI channels are generally smaller than 0.2. Some lower-level sensitive IASI channels have correlations larger than 0.3. For comparison, the interchannel correlations estimated by the HL-method are shown in Figs. $3 g-i$. The correlations estimated by these two methods generally agree well for basic correlation structures, including the nearly diagonal structures for AMSU-A, nonnegligible correlations in MHS and SAPHIR. We can also see good agreement between the BECMs in the observation space for SAPHIR/MeghaTropiques estimated by the D05 method (Fig. 3j) and that by the HL method (Fig. 3k).

The ratios of the background error SDs in the observation space estimated by the D05 method for SONDE against those used in the JMA DAS estimated by the randomization method are 0.62 for zonal wind, 0.60 for meridional wind, 0.69 for temperature, 0.57 for relative humidity, and 0.53 for surface pressure. The average value of these ratios is 0.60 , which is larger than those of $\mathrm{TBB}$ and the satellite-based $\mathrm{CNV}$, and smaller than those of nonsatellite CNV, as shown in Fig. 1. This average SDR value 0.6 is used to refine the BECM, which is used in the cycle experiments. Therefore, an analysis with $4 \mathrm{D}-$ Var using the refined $\mathbf{R}$ and $\mathbf{B}$ together, and that using the refined $\mathbf{R}$ with an inflation factor of $1 / 0.6 \cong 1.7$ in SD and the original $\mathbf{B}$ are mathematically identical. If this inflation factor is only applied to selected observation datasets, the other observation datasets get higher weight in an analysis. This value 1.7 is close to the inflation factors applied to the estimated SDs of OECMs in the previous studies, which were introduced to improve forecast accuracy and/or as the result of the adjusted eigenvalues of OECMs to improve convergence of 4DVar. The inflation factor used in Bormann et al. (2016) is 1.75, Weston et al. (2014) used about 1.65, Eresmaa et al. (2017) used 2.75, Campbell et al. (2017) used the values between 1.2 and 3.0 (this is read from their Fig. 1). Appendix A describes further consideration of the estimated BECM.

\section{b. Results of the cycle experiments}

The estimated ECMs shown in the previous subsection suggest that smaller error variances, higher density AMSU-A assimilation than CNTL, and the introduction of interchannel correlations can improve analysis and forecast accuracy. Here, we show results of the cycle experiments to clarify this suggestion.

Figure 4 shows the horizontal density of assimilated AMSU-A data in a single analysis for different thinning distances, where the AMSU-A data are assimilated at a density 5 (10) times higher than the CNTL in EXPBRT-A5 (EXP-BRT-A10).

\section{1) RESUlTS OF THE FULL ECM REFINEMENT EXPERIMENT: EXP-BR-A5}

Figure 5 shows the monthly averaged differences between the analysis fields of EXP-BR-A5 and CNTL. First, temperature at $500 \mathrm{hPa}$ changes in wide areas, and the magnitude of the changes are about $0.3 \mathrm{~K}$ (Fig. 5a). Such wide area changes in averaging fields imply changes in bias-correction values of TBB because of their wide observation coverage. The decreases of error SDs in the refined OECMs especially for TBB (Fig. 1) imply that anchor effect in EXP-BR-A5 would be weaker than those in CNTL. In fact, Fig. $5 d$ shows the bias-correction values by the VarBC scheme for AMSUA channel 6 data from MetOp-1, which is sensitive to temperature in the mid- to upper troposphere, are slightly higher (smaller in magnitude) for EXP-BR-A5 

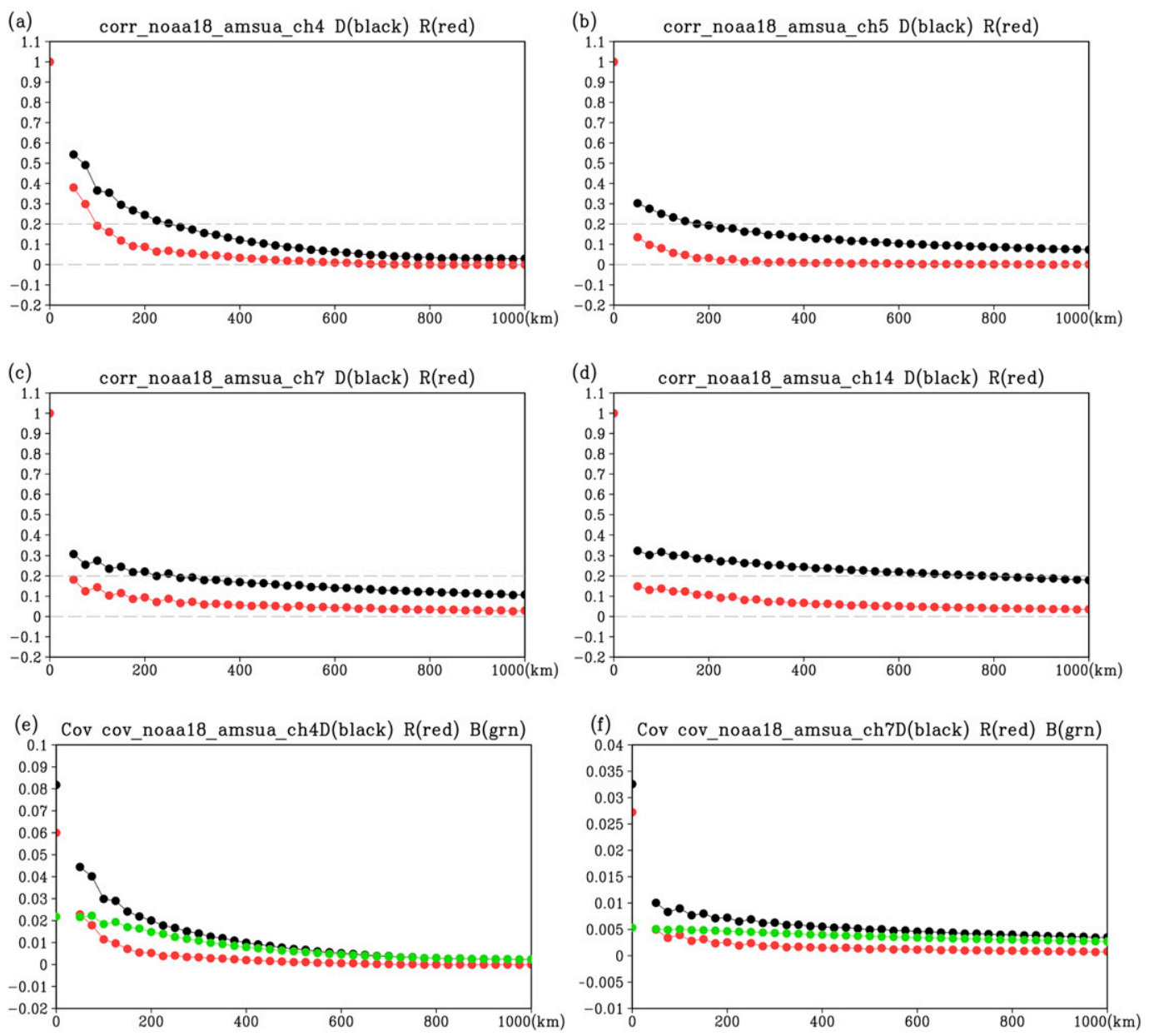

FIG. 2. Horizontal observation error correlation of AMSU-A/NOAA-18 data estimated by the D05 method: (a) channel 4, (b) channel 5, (c) channel 7, and (d) channel 14. In each panel, observation error correlations (red circles) and departure (observations minus guesses) correlations (black closed circles) are shown. The horizontal axis is distance $(\mathrm{km})$ between two observations. The vertical axis is correlation values. (e),(f) OECMs (red circles), BECMs in the observation space (green circles), and OmB covariances (black circles) for channel 4 and 7, respectively, where the vertical axis is covariance values.

than CNTL. The figure also shows that the temporal changes of the bias-correction values in these two experiments are well correlated. Therefore, despite the significant increase of TBB observation information in EXP-BR-A5, no monotonous drift of bias-correction values toward the model climate is seen. Second, Figs. $5 \mathrm{~b}$ and $5 \mathrm{c}$ show the decrease in temperature at $850 \mathrm{hPa}$ and the increase in specific humidity at $925 \mathrm{hPa}$, respectively, on the west coasts of California, Peru, and Africa. These regions correspond to the regions with large amount of low-level clouds (Teixeira and Hogan 2002), and they also correspond to the regions with large uncertainty in the analysis because accurate expression of these clouds is difficult for current NWP models (Teixeira and Hogan 2002). Since these clouds are formed under the stable layer in the planetary boundary layer, it is reasonable that the change in water vapor is accompanied by the change in temperature. On the other hand, in many other areas over sea, specific humidity decreases. These changes agree that radiance observations sensitive to low-level humidity such as GMI/GPM Core Observatory are moister (drier) than background counterparts in these moistened (dried) areas (Fig. 5f). Furthermore, midtropospheric humidity increases corresponding to humidity sounder observations (MHS, SAPHIR) that are moister than background counterparts, and this change agrees radiosonde observations and ERA5 analyses (figures not shown). In contrast, the temperature decreases over land at $925 \mathrm{hPa}$ shown in Fig. $5 \mathrm{~b}$ do not agree with radiosonde observations and other analyses such as ERA5 (figures not shown). This may imply 
(a)

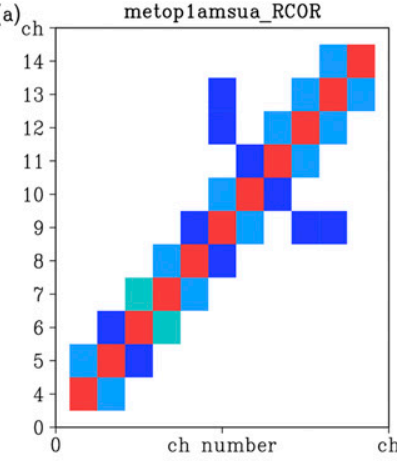

(d)

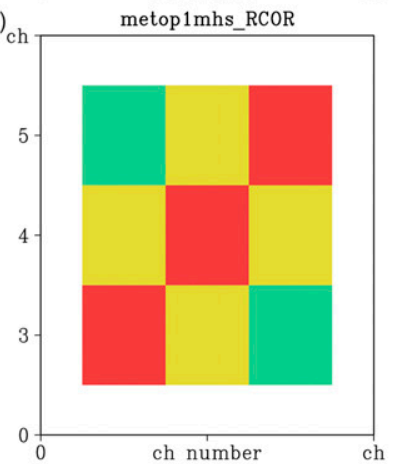

(g) HL_metop1amsua_HCorR

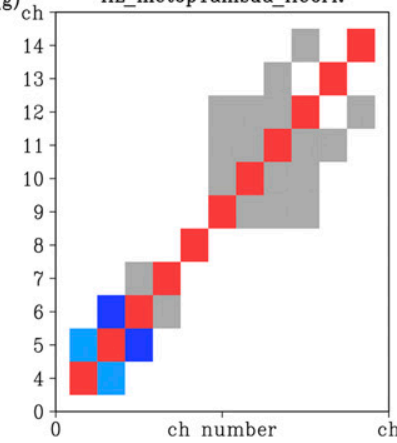

(j)

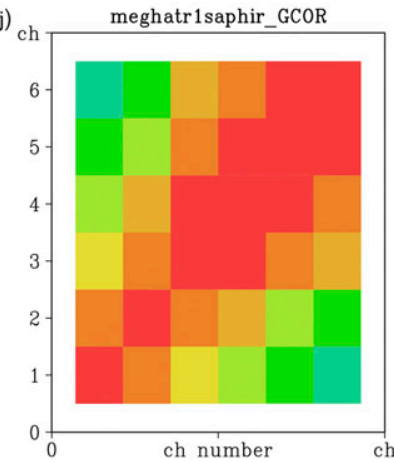

(b)

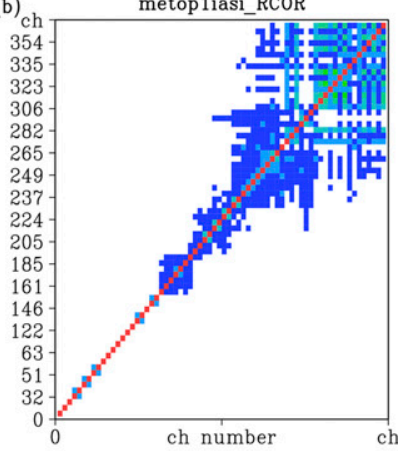

(e)

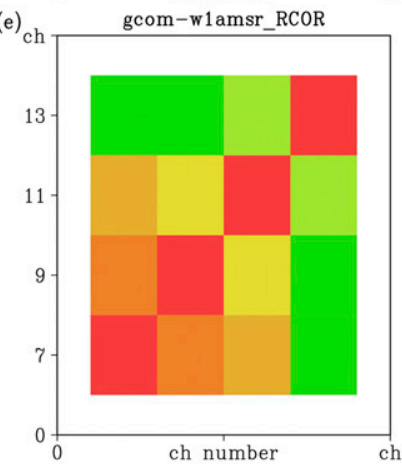

(h)
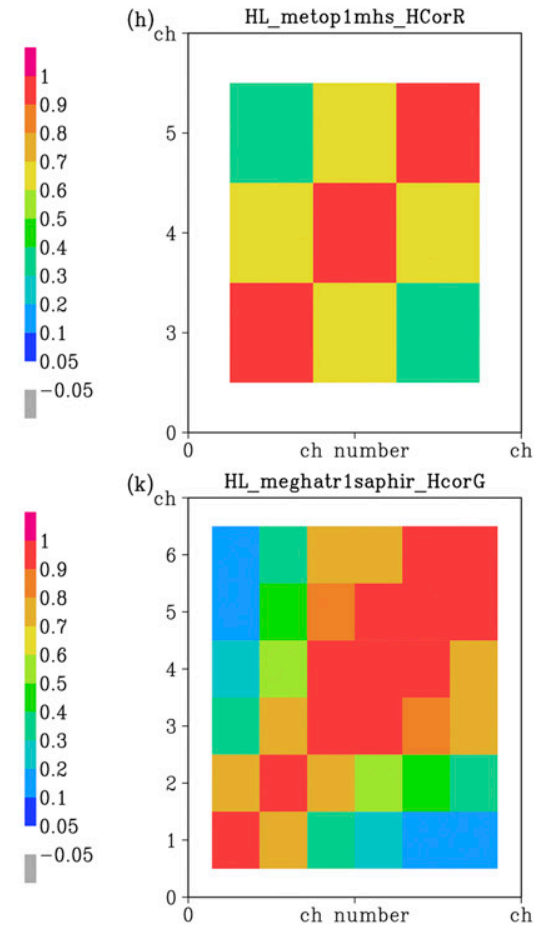

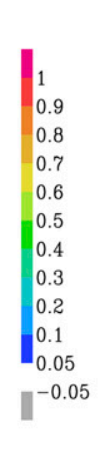

(c)

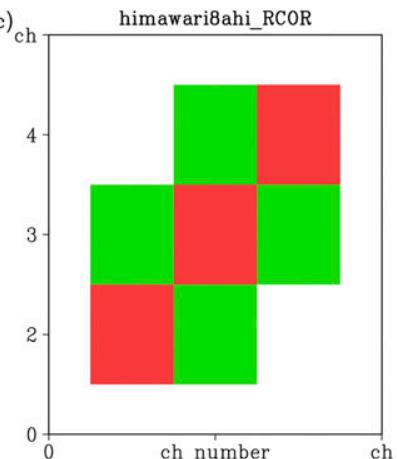

(f)

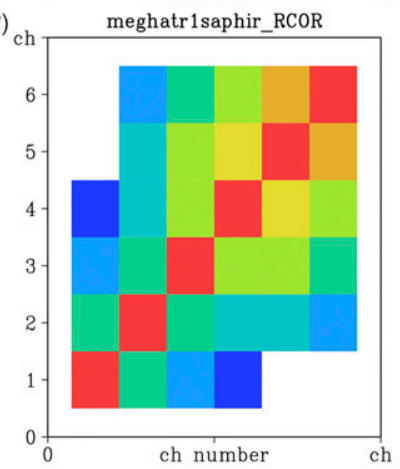

(i)
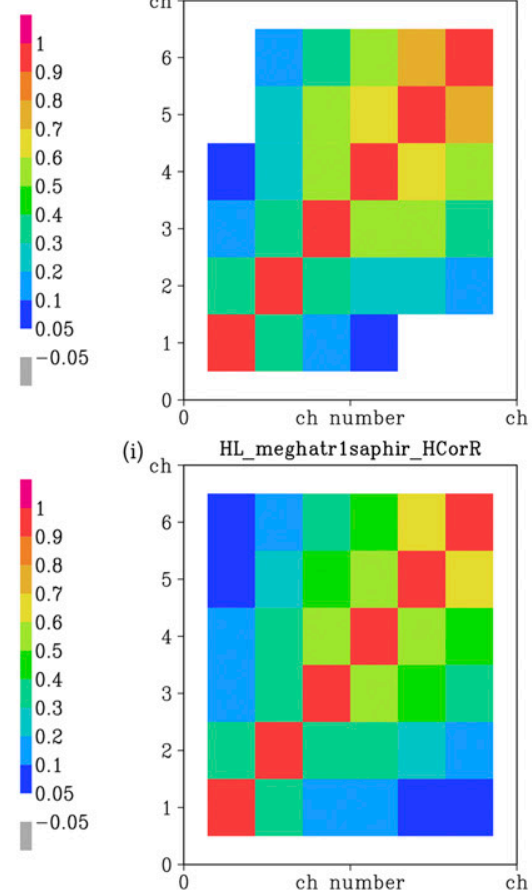
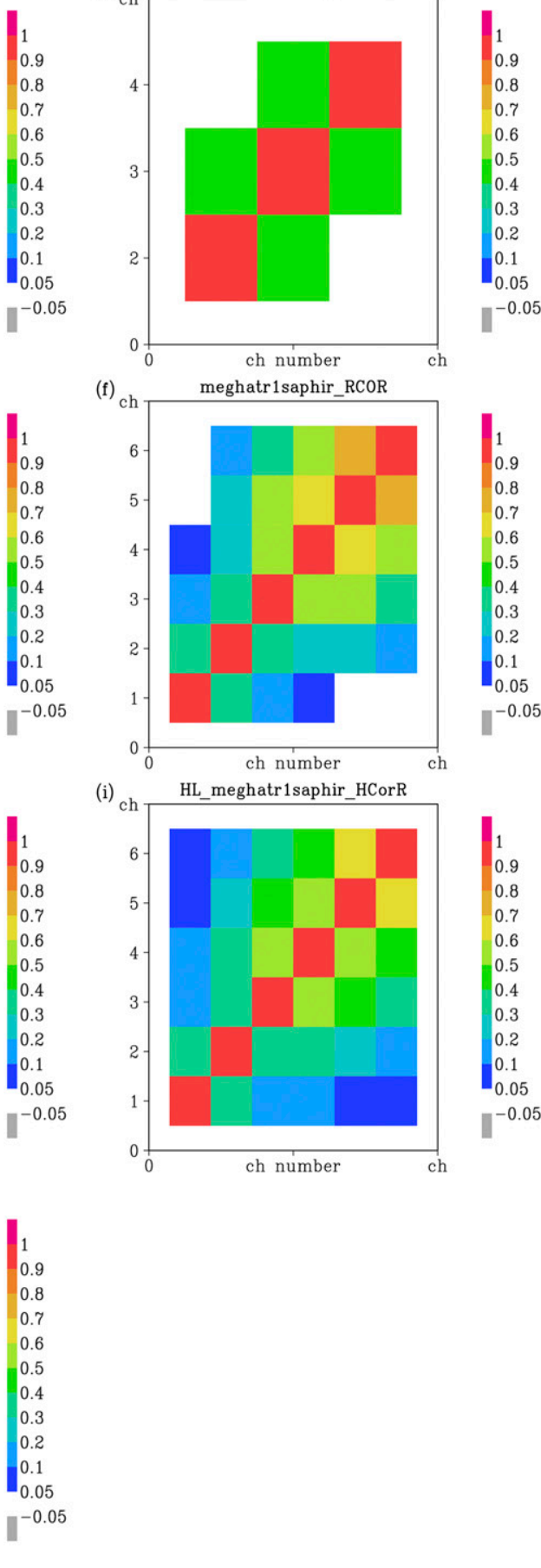

FIG. 3. Interchannel observation error correlation of radiance data estimated by the D05 method are shown (a) for AMUS-A/Met $O p$-1, (b) IASI/MetOp-1, (c) AHI/Himawari-8, (d) MHS/MetOp-1, (e) AMSR2/GCOM-W1, and (f) SAPHIR/Megha-Tropiques. For comparison, interchannel observation error correlation of radiance data estimated by the HL method are shown (g) for AMSU-A/MetOp-1, (h) MHS/MetOp-1, and (i) SAPHIR/Megha-Tropiques. The BECMs in the observation space for SAPHIR/Megha-Tropiques estimated by the (j) D05 method and by the (k) HL method are also shown. 

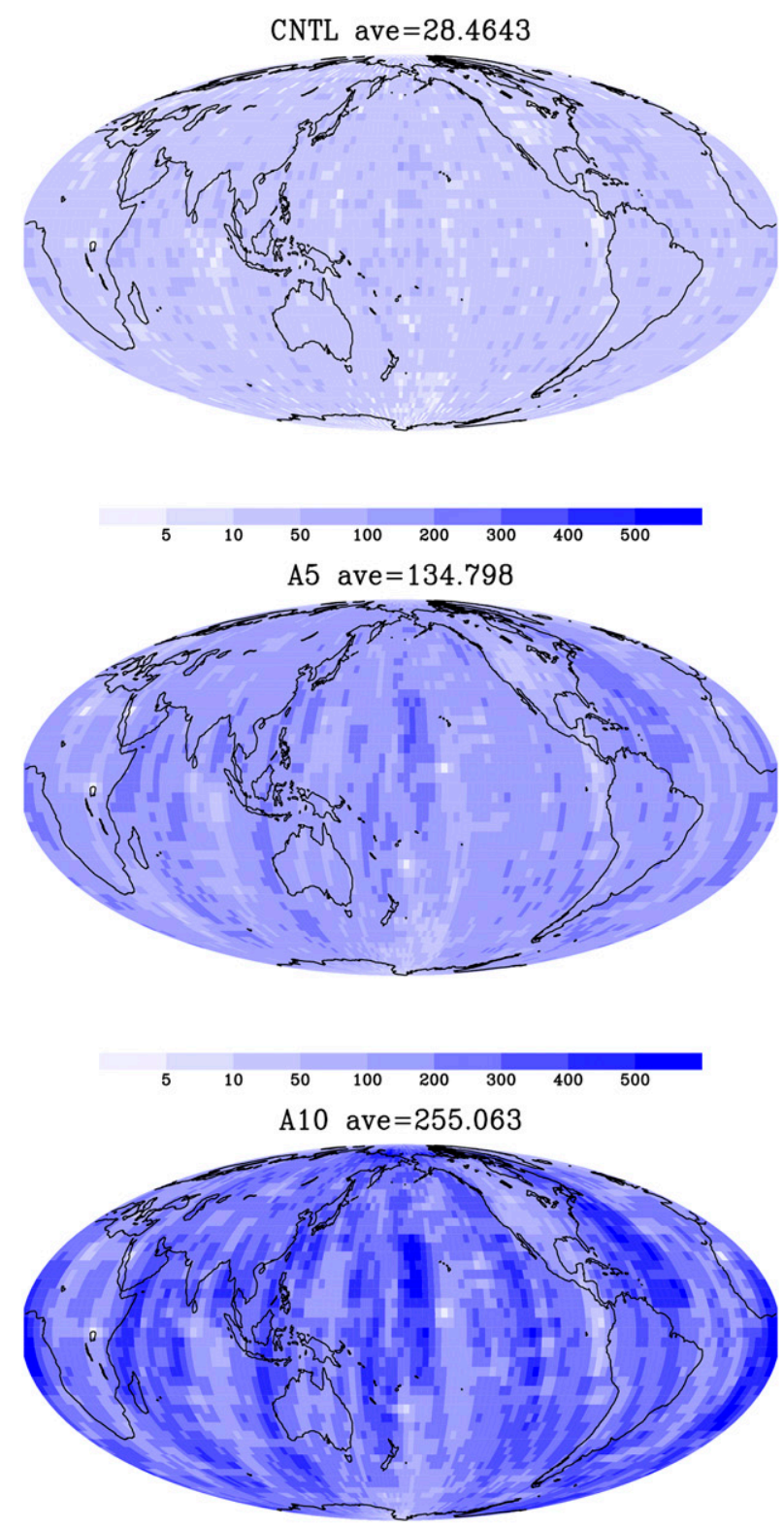

$\begin{array}{llllllll}5 & 10 & 50 & 100 & 200 & 300 & 400 & 500\end{array}$

FIG. 4. The horizontal distribution of AMSU-A data assimilated in one analysis. The analysis time is 0000 UTC 20 Aug 2016. Color shade shows the number of assimilated AMSU-A data in $3^{\circ}$ latitude by $3^{\circ}$ longitude grids. (a) CNTL, (b) EXP-BRT-A5, and (c) EXP-BRT-A10. The titles show the average assimilated data numbers in one grid.

necessity of the supplemental tuning for the refined ECMs. This point will be totally considered together with forecast accuracy changes soon later.

Figure 6 shows normalized forecast root-mean-square error (RMSE) differences for EXP-BR-A5 and CNTL, where the ERA5 analysis fields are used as truth. The normalized RMSE difference (improvement rate) is defined as

$$
\left(\mathrm{RMSE}_{\mathrm{CNTL}}-\mathrm{RMSE}_{\mathrm{TEST}}\right) / \mathrm{RMSE}_{\mathrm{CNTL}} \times 100[\%],
$$

where RMSE $\mathrm{CNTL}_{\mathrm{CN}}$ denotes the forecast RMSE of CNTL and RMSE ${ }_{\text {TEST }}$ denotes the forecast RMSE of the test experiment, which is EXP-BR-A5 here. The figure shows the forecast RMSEs of EXP-BR-A5 are generally smaller than those of CNTL up to three or four forecast days with $95 \%$ statistical significance. The largest improvement area is found in the Southern Hemisphere ( $\mathrm{SH}$, south of $\left.20^{\circ} \mathrm{S}\right)$, where the improvement rate exceeded 7\% (figures not shown). These improvements indicate that more information is properly assimilated by the refined ECMs as theoretically expected from Figs. 1 and 4. These improvements also show the validity of the estimated ECM. However, there are also some forecast accuracy degradations for the low-level tropospheric temperature in the Northern Hemisphere $(\mathrm{NH}$, north of $20^{\circ} \mathrm{N}$ ) (Fig. 6), RH in the stratosphere globally (Fig. 6), and height field and upper-tropospheric temperature in the tropics $\left(\mathrm{TP}\right.$, between $20^{\circ} \mathrm{N}$ and $20^{\circ} \mathrm{S}$; figures not shown). Although some of these degradations may be fake caused by the small forecast error growth rate, some are real degradations. Especially clear degradation is found for low-level tropospheric temperature in NH. This degradation may relate to the cold temperature bias in the lower troposphere on land found in the monthly averaged analysis of EXP-BR-A5 against CNTL (Fig. 5b). Indeed, the height field in NH also degraded. These features of forecast accuracy are also found in verifications using $\mathrm{CNV}$ as truth and $\mathrm{OmB}$ statistics (figures not shown).

These degradations found in the part of forecast RMSEs and the average field $(925 \mathrm{hPa}$ colder bias on NH land) would imply the necessity of adding supplemental tuning parameter to the estimated ECMs [section $2 \mathrm{~b}(2)$ ], such tuning may improve these degradations. Since NH is the main distribution area of the nonsatellite $\mathrm{CNV}$, and the impacts of these data are weakened in EXP-BR-A5 than those in CNTL by the refined ECMs, to enhance the impacts of these datasets is considered as the supplemental tuning. Furthermore, GPSRO can contribute this enhancement through the VarBC scheme for radiances (anchor effect). This is because the sufficiently strong anchor effect enables appropriate bias correction of the TBB and prevents bias components of the TBB from erroneously affecting the analysis field. In fact, we have already confirmed the weakened anchor effects in EXP-BR_A5 in Fig. 5d. Therefore, for the supplemental tuning to be imposed on the estimated ECMs, we can set the deflation factor 
(a) DIFF_H011_BCA5_INV-RTN_Da_T_500_1_AVE
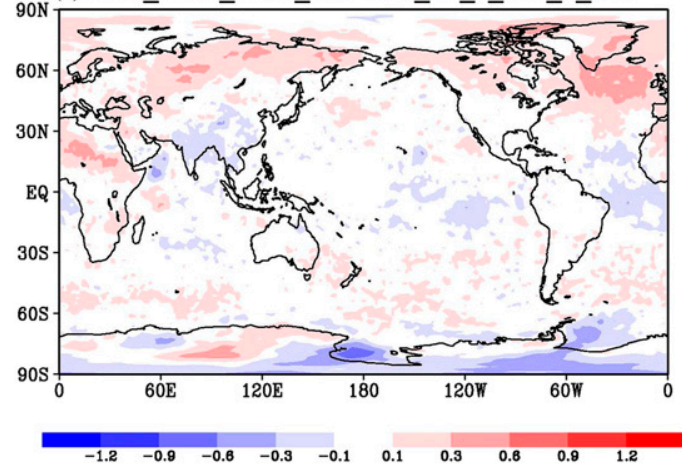

(b) DIFF_H011_BCA5_INV-RTN_Da_T_850_1_AVE

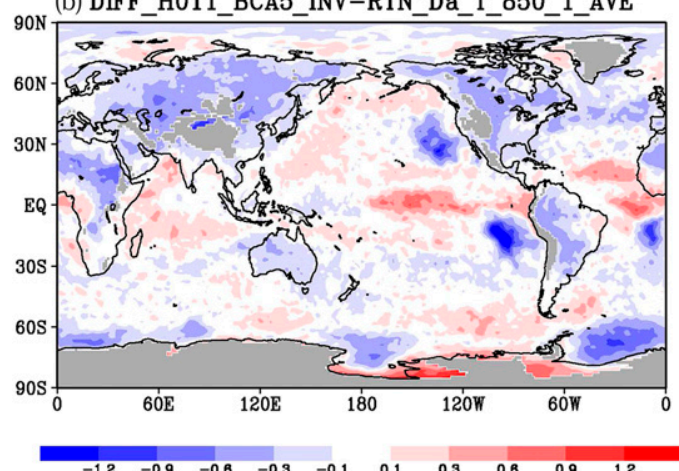

(c) DIFF_H011_BCA5_INV-RTN_Da_Q_925_1_AVE

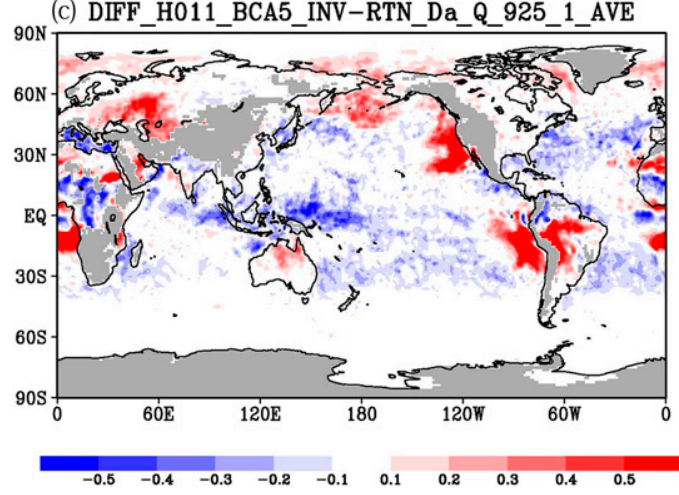

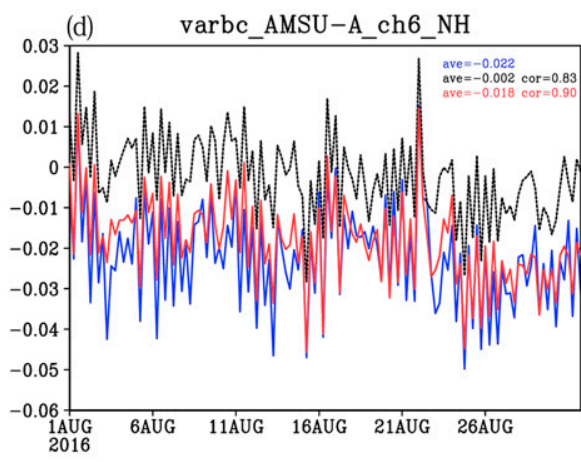
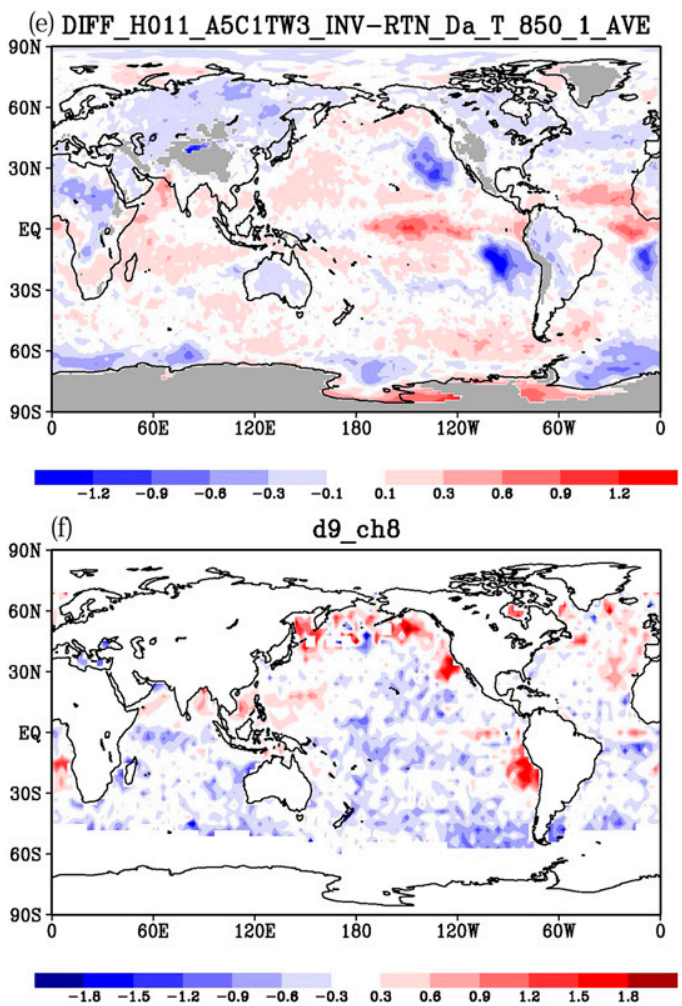

FIG. 5. Monthly averaged analysis field changes due to the ECM refinement. (left) The differences between EXPRB-A5 and CNTL for (a) temperature (K) at $500 \mathrm{hPa}$, (b) temperature (K) at $850 \mathrm{hPa}$, and (c) specific humidity $\left(\mathrm{g} \mathrm{kg}^{-1}\right)$ at $925 \mathrm{hPa}$. (d) The time sequence of the bias-correction values for channel 6 of AMSU-A/MetOp-1 by VarBC in NH for CNTL (blue line), EXP-BR-A5 (black line), and EXP-BRT-A5 (red line). In the legend, the time average during experimental period of the VarBC correction values (ave) and the correlation values with CNTL (cor) are shown. (e) The differences between EXP-BRT-A5 and CNTL for temperature (K) at $850 \mathrm{hPa}$. (f) The $\mathrm{OmB}$ values of brightness temperature $(\mathrm{K})$ for channel 8 of GMI/GPM Core Observatory.

of 0.6 for SDs of the nonsatellite CNV and GPSRO. This value is selected to be the same as the SDR for the BECM [see section $2 b(2)]$.

\section{2) RESUlTS OF THE SUPPLEMENTAL TUNING EXPERIMENT: EXP-BRT-A5}

First, let us see relative changes in EXP-BRT-A5 from EXP-BR-A5 to verify the effects of the supplemental tuning. The monthly averaged differences between the analysis fields of EXP-BRT-A5 and CNTL are generally very similar to those between EXP-BR-A5 and CNTL and the magnitudes of differences are smaller than those between EXP-BR-A5 and CNTL as shown in Fig. 5e for temperature at $850 \mathrm{hPa}$ due to the supplemental tuning parameter, which enhances the effects of nonsatellite $\mathrm{CNV}$ and GPSRO on analyses. Figure 5e shows the minus temperature biases over land in EXP-BRT-A5 are also smaller than those in EXP-BR-A5 (Fig. 5b). 

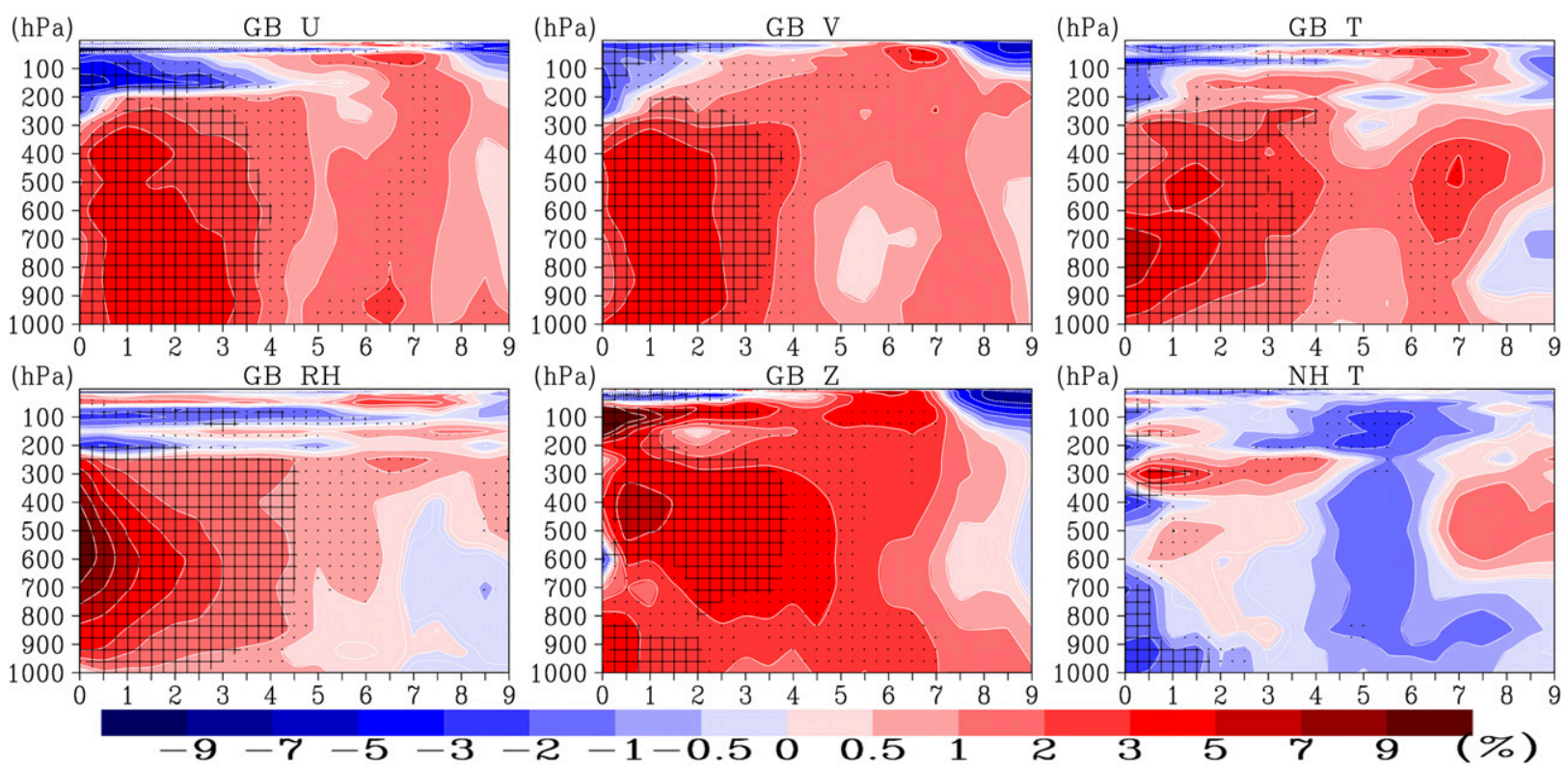

FIG. 6. Normalized forecast RMSE differences between EXP-RB-A5 and CNTL using ERA5 as truth. The normalized forecast RMSE differences averaged in the full experimental term are shown by color shades (\%), where red (blue) shows EXP-RB-A5 has smaller (larger) forecast RMSE than CNTL. The cross hatches (dots) denote 95\% (68\%) statistical significance. The vertical axis is pressure height in $\mathrm{hPa}$. The horizontal axis is the forecast time 0-9 days. (top) The global averaged normalized RMSE differences for (top) (from left to right) $U, V$, and $T$, and (bottom) (from left to right) RH, height ( $Z$ ), and the NH averaged normalized RMSE differences for $T$.

We can also see in Fig. 5d that VarBC correction values are much close to CNTL in comparison with EXT-BRA5. These results clearly show that the anchor effects of observation are enhanced by the supplemental tuning.
Figure 7 shows the forecast RMSE improvement rate of EXP-BRT-A5 against EXP-BR-A5, where the ERA5 analysis fields are used as truth. We can find that forecast RMSEs of EXP-BRT-A5 are generally smaller than those of EXP-BR-A5 up to three or four forecast
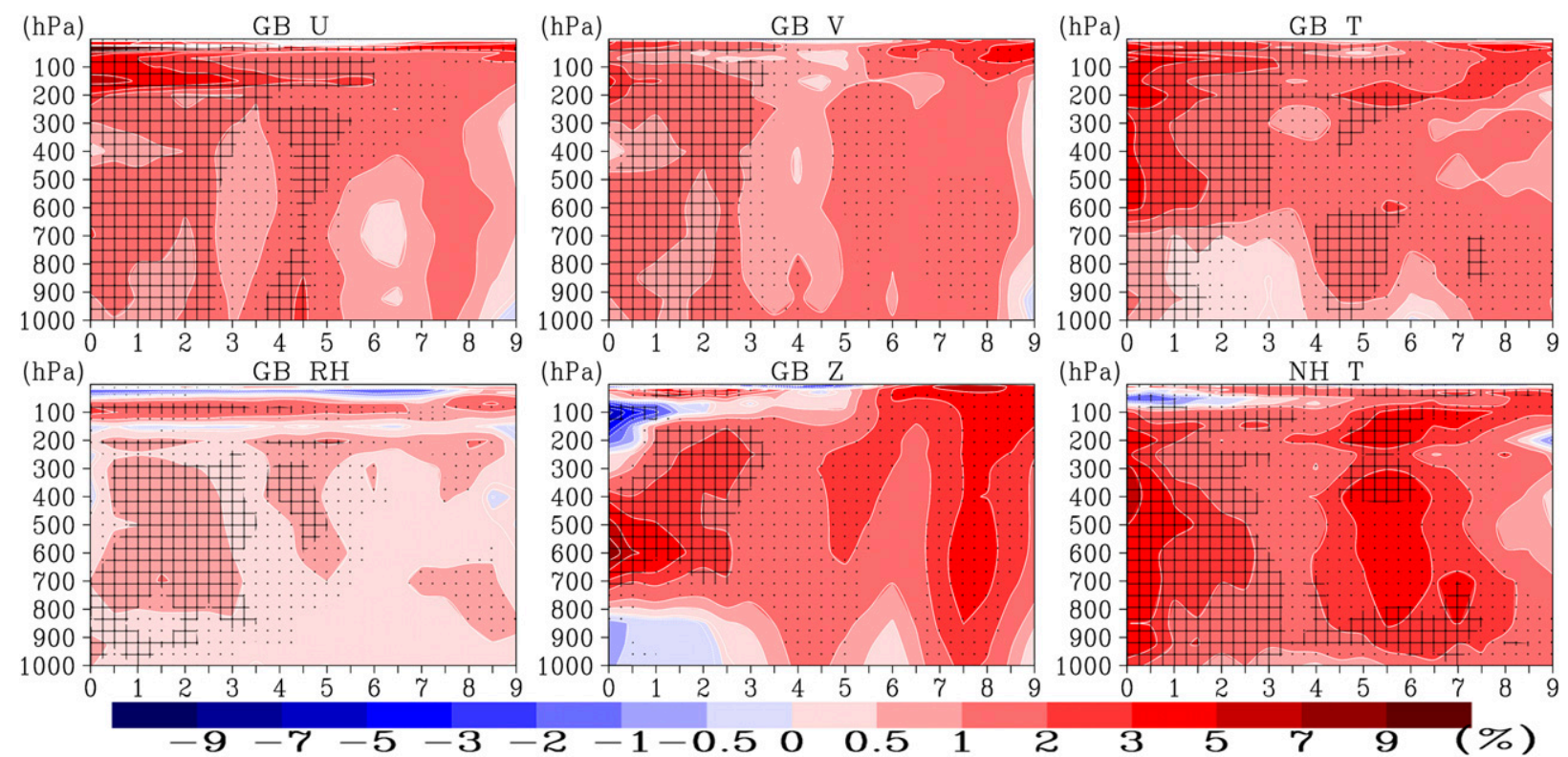

FIG. 7. As in Fig. 6, but that normalized forecast RMSE differences between EXP-BRT-A5 and EXP-RB-A5 are shown, where red (blue) shows EXP-BRT-A5 has smaller (larger) forecast RMSE than EXP-BR-A5. 

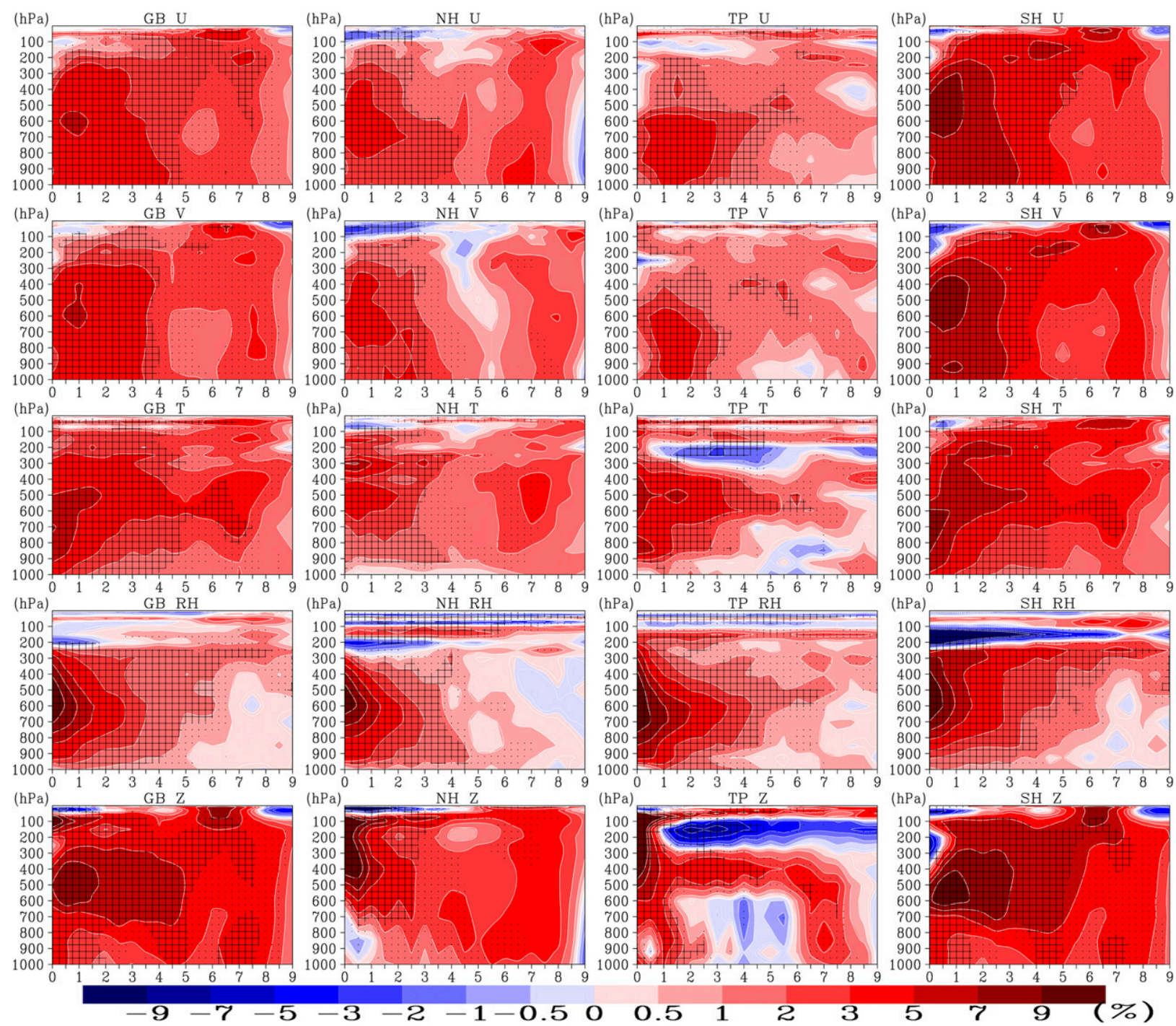

FIG. 8. As in Fig. 6, but that the differences between EXP-BRT-A5 and CNTL are shown, and that the four columns show (from left to right) the global (GB), NH, TP, and $\mathrm{SH}$, and (from top to bottom) $U, V, T, \mathrm{RH}$, and $Z$. Red (blue) shows EXP-BRT-A5 has smaller (larger) forecast RMSE than CNTL.

days with $95 \%$ statistical significance. Especially, the temperature forecasts in $\mathrm{NH}$, where the explicit degradation is found in EXP-BR-A5 (Fig. 6) are statistically significantly improved up to 7 days. Therefore, the supplemental tuning parameter works well.

Second, let us see changes in EXP-BRT-A5 from CNTL finely to verify the full effects of the ECM refinement with the supplemental tuning parameter. Figure 8 shows the forecast RMSE improvement rate of EXP-BRT-A5 against CNTL, where ERA5 analysis fields are used as truth. We can find that forecast RMSEs of EXP-BRT-A5 are generally smaller than those of CNTL up to four or five forecast days with $95 \%$ statistical significance. The largest improvement area is $\mathrm{SH}$, where the improvement rate exceeds $9 \%$. This distribution is explained by the fact that TBB data, which are given significantly smaller error SDs by the D05 method, are more important in $\mathrm{SH}$ than $\mathrm{NH}$ due to lack of direct observations. Another possible reason is more active baroclinic instability in $\mathrm{SH}$ since this is a boreal summer experiment. However, this is not the main reason because this distribution is also found in the winter experiment EXP-BRT-A5-WIN (figures not shown).

In Fig. 8, some minor forecast accuracy degradations are also found for the RH fields above $200 \mathrm{~Pa}$, for the height fields in tropics, and for the upper-tropospheric temperature in TP. Here, we consider these degradations. First, since the RH degradation is also found in 

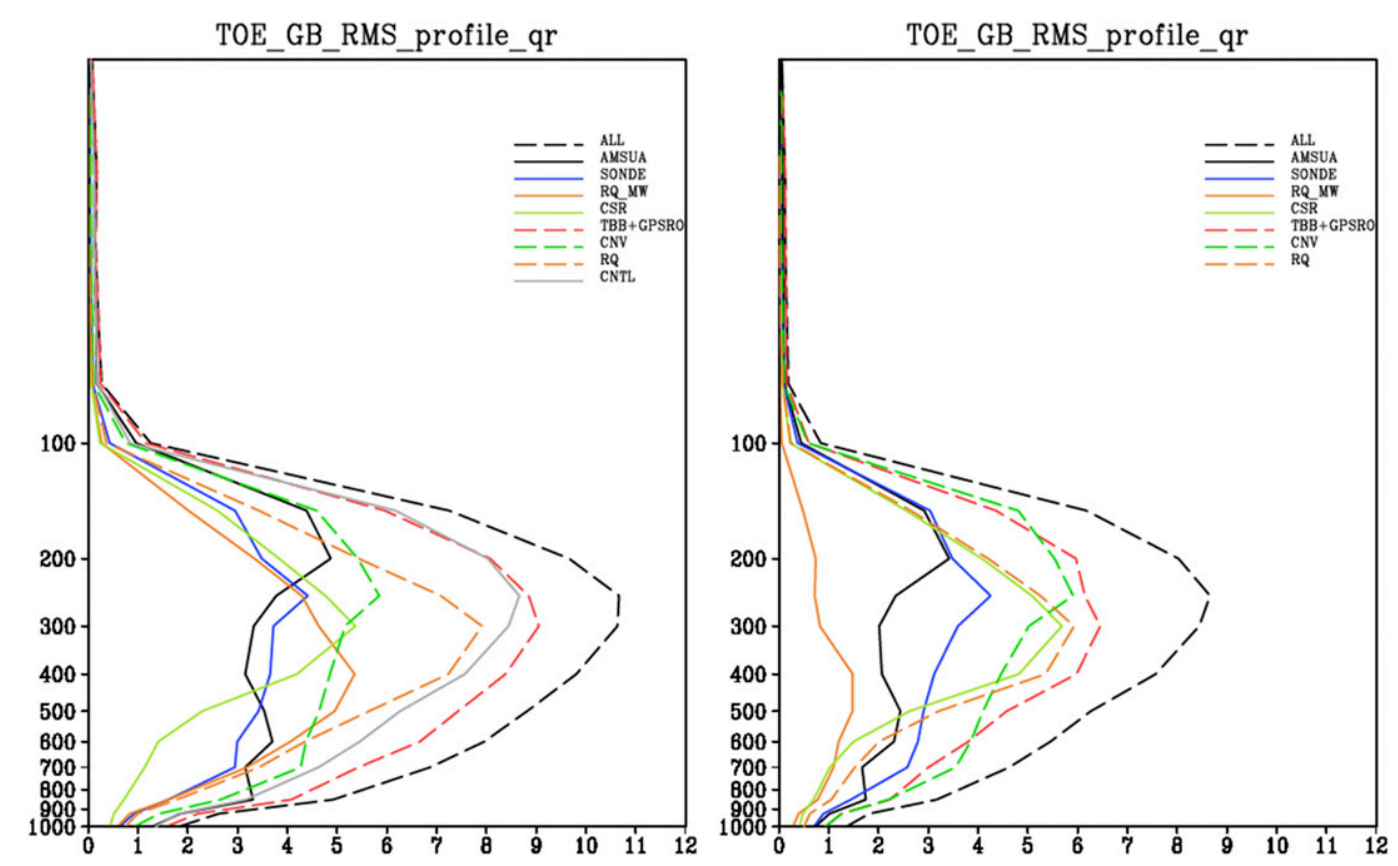

FIG. 9. Contribution of each observation dataset to the analysis field of specific humidity for the randomly selected single analysis at 0000 UTC 12 Aug 2016. The contribution is estimated as analysis increments generated by each observation dataset (partial analysis increment vector, PIV) estimated by the TL-based observation impact estimation method (Ishibashi 2011). The global root-mean-square (RMS) averages of the PIV of each observation dataset are shown for (left) EXP-BRT-A5 and (right) CNTL, respectively, where PIVs are normalized by the average specific humidity of the background field in each pressure level. The dataset names in the legend are the same as those in Table 1 and Fig. 1, except that ALL and RQ_MW denote the total analysis increments and the total PIV of the microwave radiances in RQ, respectively. The solid gray line in the left panel show the ALL of CNTL for comparison. The vertical axis denotes pressure height $(\mathrm{hPa})$, the horizontal axis denotes RMS average values of PIVs.

specific humidity field (figures not shown), this degradation comes from not temperature but specific humidity. To check specific humidity analyses, Fig. 9 shows the analysis increment magnitudes generated by each observation dataset, which are calculated by the tangent linear approximation-based observation impact estimation method (Ishibashi 2011), for a single analysis at 0000 UTC 12 August. We can see that the specific humidity analysis increments above $200 \mathrm{hPa}$ increase, where the contributions from TBB including humiditysensitive sensors clearly increase. Therefore, the RH forecast field changes are caused by the ECM refinement. Figure 9 also shows the increase of the specific humidity analysis increments for the humidity-sensitive microwave radiances as expected from Fig. 1. On the other hand, Fig. 10 shows forecast RMSE growth rates of $\mathrm{RH}$ at $150 \mathrm{hPa}$ is smaller than those of the $500 \mathrm{hPa}$ height field (Z500) in SH. This means any forecast error verifications for upper-level $\mathrm{RH}$ is more difficult than those for $\mathrm{Z} 500$ in $\mathrm{SH}$ due to this smallness of error growth rate since any verification is based on smallness of error of reference analyses in comparison with forecast error differences of two experiments. Figure 10 also shows that the error growth rate is also small for Z500 in TP. The error growth rates for the upper-tropospheric $Z$ and $T$ in TP are also small (figures not shown). These agree with small variability of the height field in TP. Furthermore, we can also see the error growth rate of $\mathrm{Z} 500$ in $\mathrm{SH}$ detected by the verification using $\mathrm{CNV}$ as truth is also small. Therefore, the CNV verification is more difficult than the ERA5 verification in this context.

Figure 11 is the same as Fig. 8, except that CNV is used as truth. The forecast RMSEs of EXP-BRT-A5 are generally smaller than those of CNTL. In SH, forecast RMSEs are clearly reduced by the refined ECM. In NH and TP, winds and midlevel humidity are also clearly improved. Whereas, other parts are generally neutral, the degradations in the height fields mainly in TP are shown. These results of the CNV verification agree with the ERA5 verification if we consider the smaller error growth rates for height field in TP and the smaller error growth detection rate by the $\mathrm{CNV}$ verification described in previous paragraph. Figure 12 shows the OmB statistics. We can see the SDs of OmB in EXP-BRT-A5 are generally smaller than those in CNTL. In the stratosphere, some degradations are seen for radiosonde $\mathrm{RH}$ 


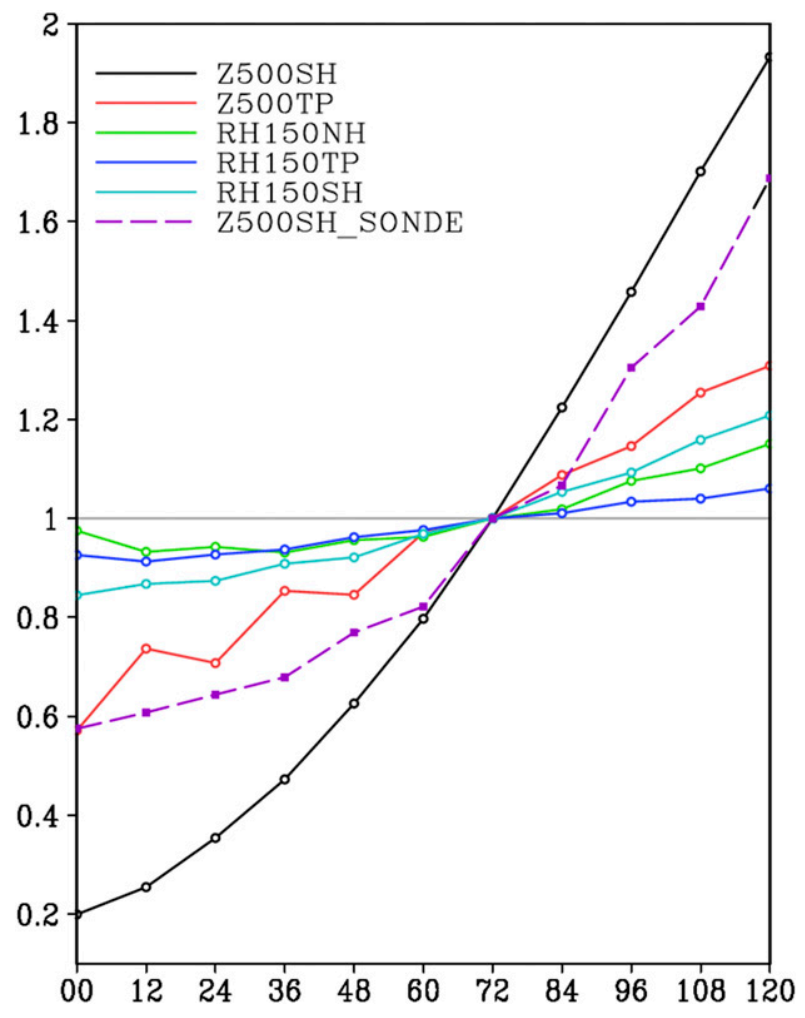

FIG. 10. Forecast error growth rates. This figure shows the growth of the forecast RMSEs during the initial time to 5 days for selected variables, areas, and pressure heights, where the forecast RMSEs are normalized by those of the 3-day forecasts. The monthly averages for August 2016 in CNTL are shown. The vertical axis denotes the normalized forecast RMSE values, and the horizontal axis shows forecast time (hour). The black and the red lines show the RMSEs of $Z$ at $500 \mathrm{hPa}$ in SH and TP, respectively. The green, blue, and aqua lines show the RMSEs of RH at $150 \mathrm{hPa}$ in NH, TP, and SH, respectively. These RMSEs are calculated using ERA5 as truth. The purple broken line shows the RMSEs of $Z$ at $500 \mathrm{hPa}$ in $\mathrm{SH}$, where CNV data are used as truth.

and GPSRO in $\mathrm{NH}$ and $\mathrm{SH}$. These results of the $\mathrm{OmB}$ verification agree with the ERA5 verification.

3) RESULTS OF THE ADDITIONAL EXPERIMENTS: EXP-BR-A1, EXP-BRT-A1, EXP-BRT-A10, AND EXP-BRT-A5-WIN

Figure 13 shows the forecast RMSE improvement rates of the additional experiments: EXP-BR-A1, EXPBRT-A1, EXP-BRT-A10, and EXP-BRT-A5-WIN using the ERA5 data as truth. First, Figs. 13a and 13b show the forecast RMSEs of EXP-BR-A1 and EXP-BRT-A1 are statistically significantly smaller than those of CNTL. Therefore, only the use of the refined ECMs without the high-density assimilation of AMSU-A can improve forecast accuracy. The improvement rate for EXP-BRT-A1 is larger than EXP-BR-A1 as the same as EXP-BRT-A5 is better than EXP-BR-A5 (Fig. 7).
Therefore, the supplemental tuning parameter is also adequate for the refined ECM without the high-density assimilation of AMSU-A. Second, Figs. 13d and 13e show the forecast RMSE improvement rates for EXPBR-A5 against EXP-BR-A1, and for EXP-BRT-A5 against EXP-BRT-A1, respectively. We can see the forecast accuracy improvement by increasing AMSU-A density is larger in EXP-BRT-A5 than in EXP-BR-A5. This means the refined ECMs with the supplemental tuning parameter can assimilate more observation information. Figure 13f shows the forecast accuracy of EXP-BRT-A10 is no better than EXP-BRT-A5, where clear degradation is seen in the middle troposphere to the lower stratosphere within 2 or 3 days forecasts. Suppose this perturbation is the uncertainty of the estimated MCD, this result shows the sensitivity of the forecast accuracy to the MCD uncertainty. However, the accuracy of EXP-BRT-A10 is still statistically significantly better than CNTL (figures not shown). This result show robustness of the MCDs estimated by the D05 method. Finally, Fig. 13c shows the forecast RMSE improvement rates for EXP-BRT-A5-WIN against CNTL-WIN. We can see clear forecast accuracy improvement. This result shows ECMs diagnosed by the D05 method using summer data work well in winter, therefore, seasonal dependence of estimated ECMs are small. This result shows robustness of the D05 estimation.

\section{Summary and conclusions}

In this study, we estimated ECMs for all observations and background variables by combining multiple ECM estimation methods (the D05 method supported by the HL method and the randomization method). In addition, we verified the impacts of the estimated ECMs on the accuracy of global NWP using cycle experiments on the JMA global NWP system. The ECM diagnostics results have shown the following: 1) the diagnosed error variances are generally much smaller than the current operational settings for almost all observation types and background field variables, especially for remote sensing observation data; 2) interchannel correlations of observation errors of water vapor sensitive radiance data are not negligible; and 3) the MCDs of AMSU-A data are around $50 \mathrm{~km}$ except for channel 4, whose correlation distance is about $100 \mathrm{~km}$. These diagnosed results agree with those in the previous studies (Bormann and Bauer 2010; Bormann et al. 2010, 2013; Stewart et al. 2014; Weston et al. 2014; Campbell et al. 2017; Bormann et al. 2016).

Second, the cycle experiments were conducted to evaluate the effects of introducing these new ECMs on 

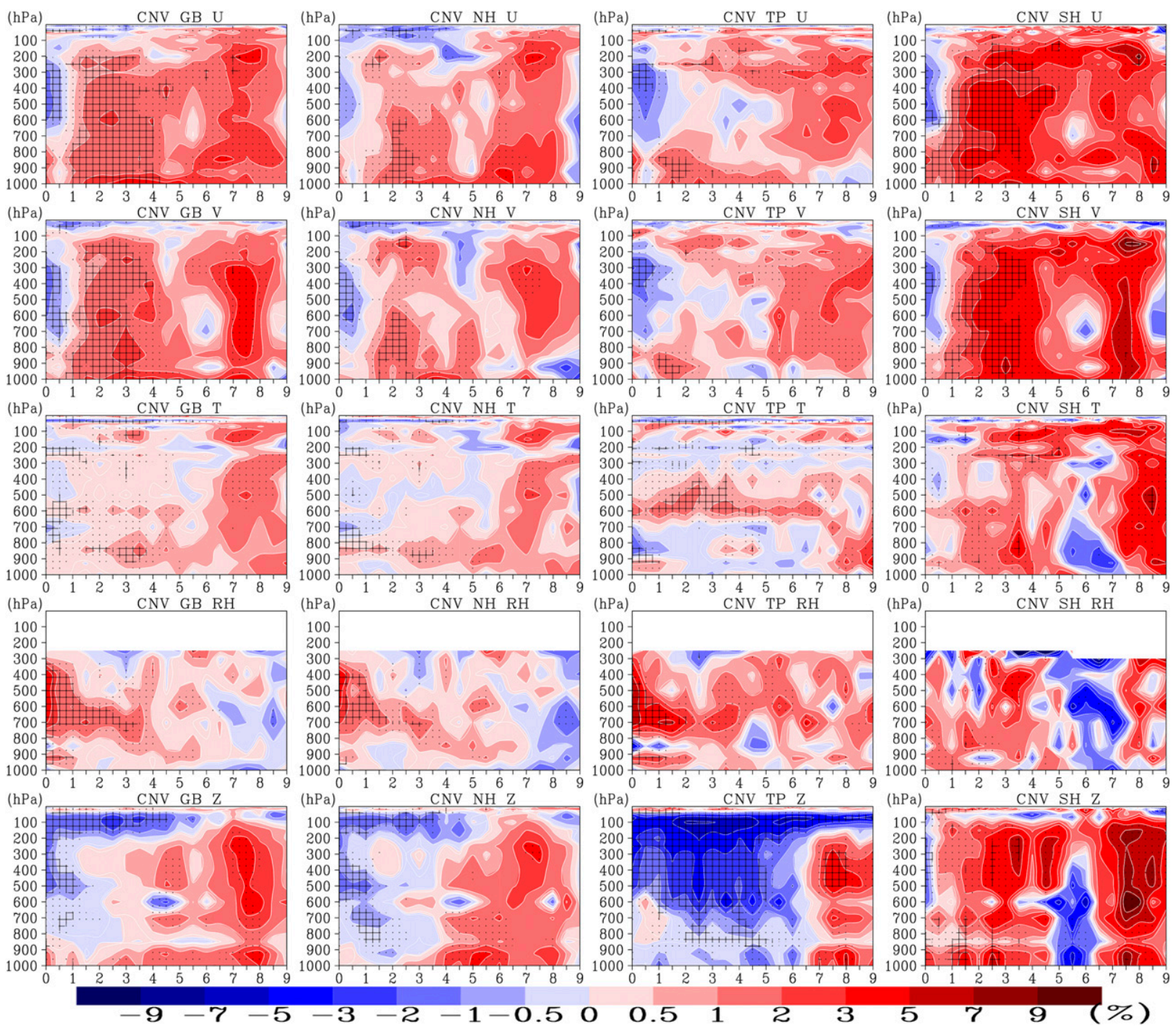

FIG. 11. As in Fig. 8, but that conventional observation data are used as truth.

the JMA global NWP system, where two main test experiments and four additional test experiments are included. The results of the main experiments have revealed the following: 1 ) introducing the diagnosed ECMs generally statistically significantly improves forecast accuracy compared to CNTL even when no supplemental tuning is applied (EXP-BR-A5); 2) the supplemental tuning parameter, which is the deflation factor with the value of 0.6 in SD for nonsatellite conventional observations and GPSRO, imposed on the estimated ECMs statistically significantly improve forecast accuracy (EXP-BRT-A5); 3) this value 0.6 is determined as the same value as the ratio of the refined SD to the original SD of background errors; 4) the number of the tuning parameter one in this study is much smaller than that in a data assimilation system with ECMs given by empirical tunings. The results of the additional experiments have revealed the following: 1) the result of the EXP-BRT-A10 experiment that assimilated AMSU-A data with a 10 times higher density and a thinning distance of $80 \mathrm{~km}$ still showed improved accuracy over CNTL; however, it tended to be no better than EXP-BRT-A5; 2) the ECMs estimated using summer data can improve forecast accuracy in winter, which denotes robustness of the estimated ECMs (EXPBRT-A5-WIN); and 3) pure ECM refinement without thinning distance refinement can also improve forecast accuracy (EXP-BR-A1, EXP-BRT-A1).

Finally, limitations of this study and plans for future studies to address these limitations include the following aspects. First, in this study, only interchannel observation error correlations of radiance data were introduced. 
(a)

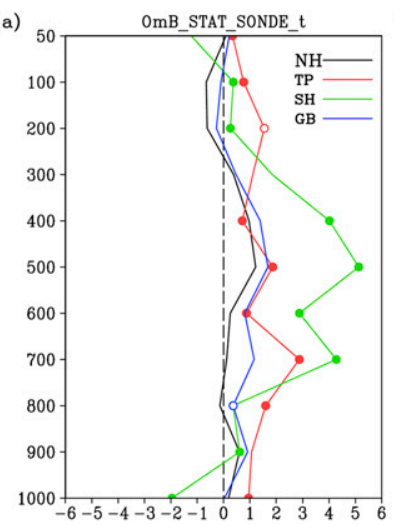

(e)

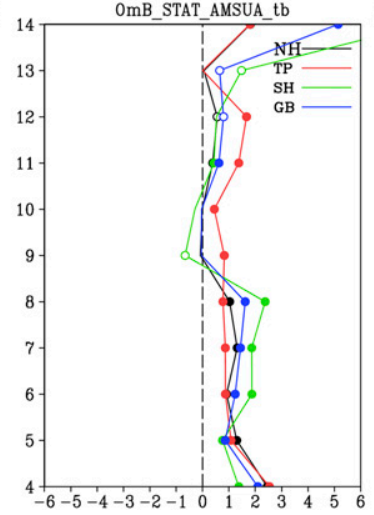

(b)

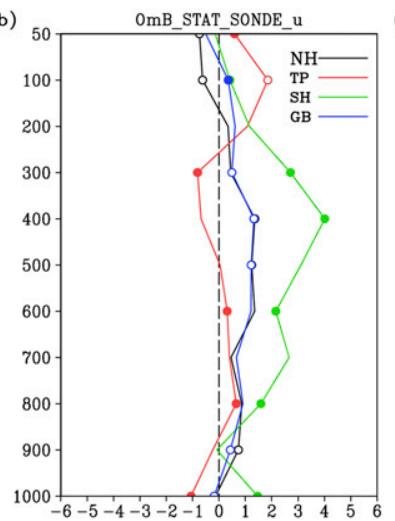

(f)

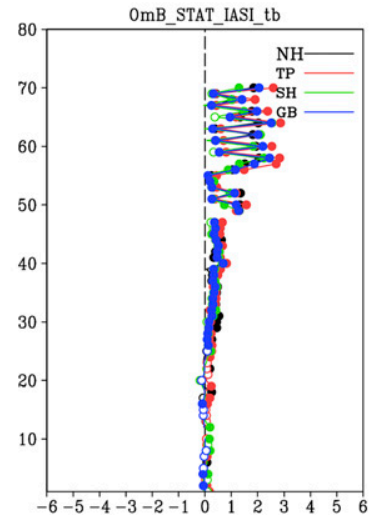

(c)

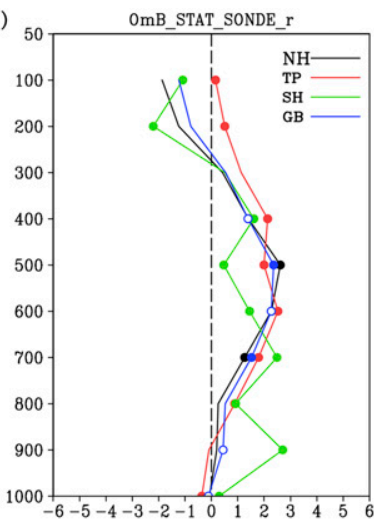

(g)

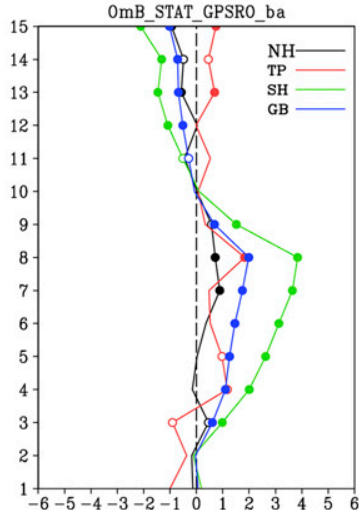

(d)

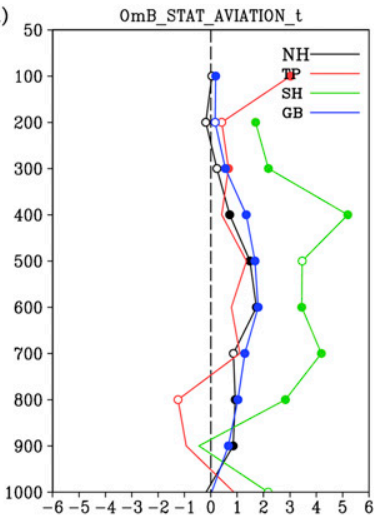

(h)

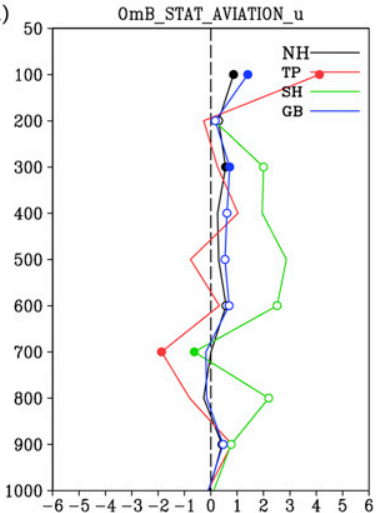

FIG. 12. Normalized OmB SD differences between EXP-BRT-A5 and CNTL. The normalized OmB SD differences averaged in the full experiment term are shown for NH (black lines), TP (red lines), SH (green lines), and GB (blue lines), where plus (minus) values show EXP-BRT-A5 has smaller (larger) SDs than CNTL. The closed (open) circles denote $95 \%$ (68\%) statistical significance. (a) SONDE T, (b) SONDE U, (c) SONDE RH, (d) AVIATION T, (e) AMSU-A TBB, (f) IASI TBB, (g) GPSRO BA, and (h) AVIATION U. The horizontal axis denotes the normalized OmB SD difference values (\%). The vertical axis denotes height in $h P a$ for (a)-(d), and (h), channel numbers for (e) and (f), height in $\mathrm{km}$ for (g).

Therefore, studies on observation error correlations in space-temporal directions including other observation datasets are needed. Furthermore, introducing these correlations into a DAS requires research on efficient processing of nondiagonal OECMs in 4D-Var. This requires efficient computation across computation nodes (nonlocal computation) for OECM inverse computation and cost function and its gradient computation. Although the observation space 4DVar does not require the OECM inverse as long as preconditioning using only diagonal elements of OECM is enough (Campbell et al. 2017), such more extensive introduction of observation error correlations may result in a loss of this advantage. Furthermore, Bormann et al. (2011) showed interchannel and horizontal correlation structures depend on the atmospheric state (clear, cloudy, and rainy). Studies on nondiagonal and flow dependent OECMs with nonlocal calculations are needed.

Second, this study requires one supplemental tuning parameter to enhance NWP improvement. Inaccuracy of the BECM derived by the NMC method would be one possible reason for the need for this parameter. Therefore, studies on DASs with fully flow-dependent BECM such as ensemble-based 4DVar DASs are needed. Since any current ensemble-based DASs also includes inflation factors of ensemble spreads as tuning parameters, these studies are also one of the main themes of studies of ensemble-based DASs. These studies will make it possible to assimilate more observation information in the DAS, and improve NWP accuracy. Furthermore, although, in this study, the supplemental tuning was simply done as deflation of variances estimated by the D05 method, more sophisticated approach to improve ECMs estimated by the D05 method may be possible by adding extra information. Adding additional information to sample ECMs to construct accurate ECMs is an important research theme for both OECMs and BECMs. For all such future studies, ECM estimation of all observation and background variables by the D05 method and their use in a NWP system here is essential starting points, since it prevents failure caused by adjusting only part of data. 

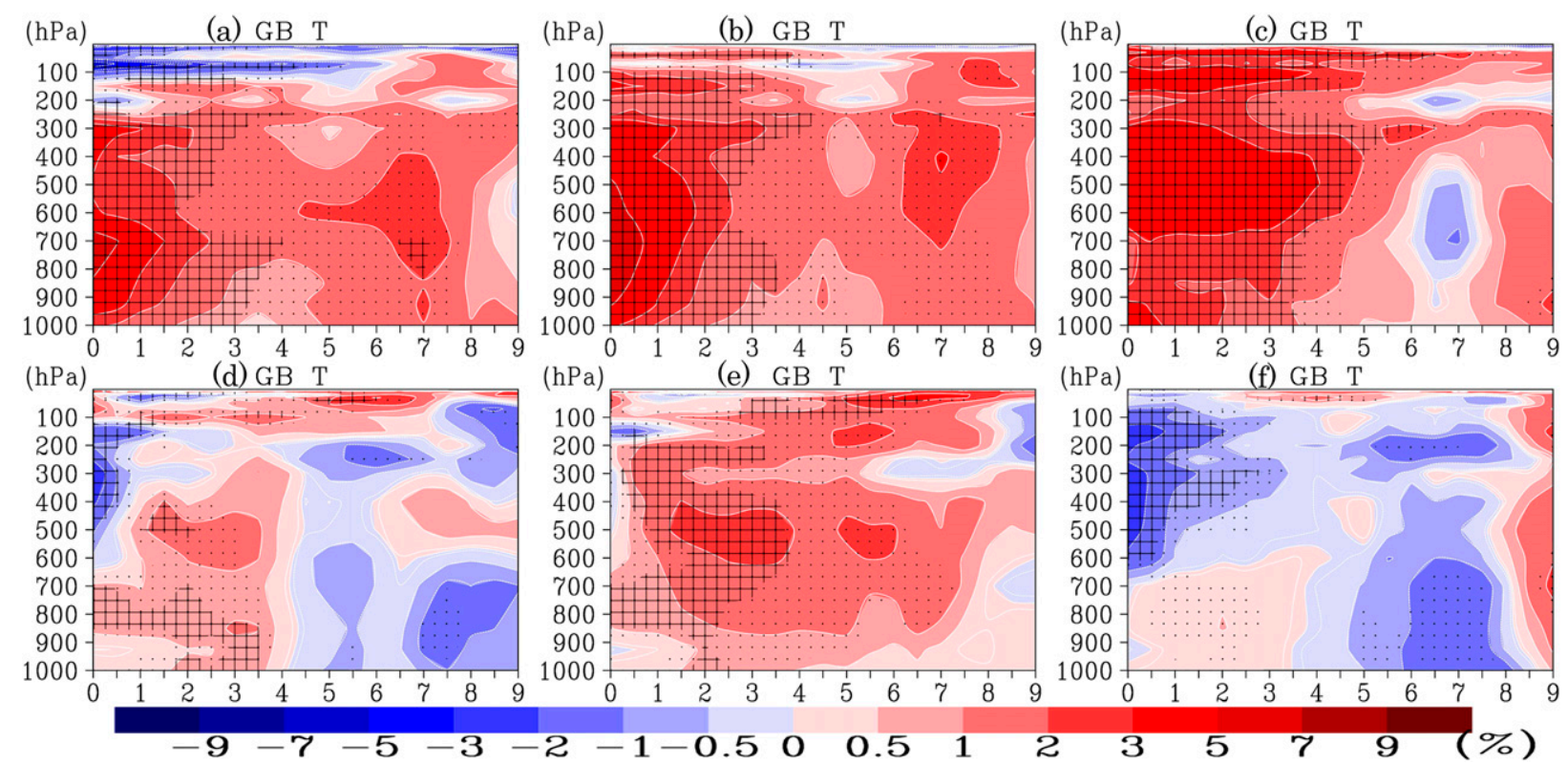

FIG. 13. Normalized forecast RMSE differences for the additional experiments. As in Fig. 6, but for following points. All panels show the global averaged normalized RMSE differences for $T$. (top) (from left to right) The differences for (a) EXP-BR-A1 against CNTL, (b) EXP-BRT-A1 against CNTL, (c) EXP-BRT-A5-WIN against CNTL-WIN. (bottom) (from left to right) The differences for (d) EXPBR-A5 against EXP-BR-A1, (e) EXP-BRT-A5 against EXP-BRT-A1, and (f) EXP-BRT-A10 against EXP-BRT-A5. In each panel, red (blue) shows the former experiment has smaller (larger) RMSE than the latter experiment.

Acknowledgments. The author is grateful to all those individuals who contributed to developing the JMA global NWP system and to the four anonymous reviewers. I also thank my colleagues for discussions and support in various situations. This work was supported by JSPS KAKENHI Grant JP17K05658.

\section{APPENDIX A}

\section{Validity and Meanings of the Estimated BECM}

Here, we consider the validity of the estimated SDR of the BECM shown in section 4. First, this estimation is based on the assumption that SONDE distribution and BECM SDs are homogeneous. Therefore, this estimation includes errors of these assumptions. Second, although, the rescaling factor used in the NMC method, which converts 24-h forecast error differences into 6-h forecast error, is set to 0.9 for SDs in the JMA system (JMA 2013), this value has not been definitively determined from theory or experiments. This is one of the main limitations of the NMC method (Bannister 2008). The values smaller than 1 such as 0.6 here are not excluded by the previous studies on forecast error growth (Lorenz 1982; Dalcher and Kalnay 1987; Reynolds et al. 1994; Žagar et al. 2017). Third, Errico et al. (2015) showed the ratio is smaller (larger) than 1 in the extratropic (tropical) regions in their observation system simulation experiment, and we can see it would be smaller than 1 in global average from their Fig. 1. Fourth, although this rescaling factor value of 0.9 is the same as Rabier et al. (1998), observation data have much increased from 1998 in these 20 years. Therefore, if such a large rescaling factor of 0.9 was due to a shortage of observation data (Rabier et al. 1998), the rescaling factor should be smaller than 0.9 in the current 4DVar DAS.

Finally, let us consider meanings of closeness of the reciprocal value of the SDR of BECM (0.6), which is 1.7, to the inflation factors applied diagnosed $\mathbf{R}$ in the previous studies (see the explanation of estimated BECM in section 4). This closeness may show that the origin of these inflation of $\mathbf{R}$ is the largeness of $\mathbf{B}$ used in DASs. In fact, in these studies, except for Bormann et al. (2016), the BECMs are basically derived by climatological method such as the NMC method. Bormann et al. (2016) used the ensemble of DASs (Bonavita et al. 2012), where the inflation factor of around 1.5 is applied to the raw ensemble-based BECM to enlarge the SDs. Since this inflation factor is determined from the ratio of the forecast error SD and forecast ensemble spread, it seems that there is no ambiguity. However, if the smallness of the spread is caused by smallness of degrees of freedom to capture leading singular vectors, namely the smallness of error growth rate represented by the ensemble, then the SDs of the BECM in their DAS would be too large. 


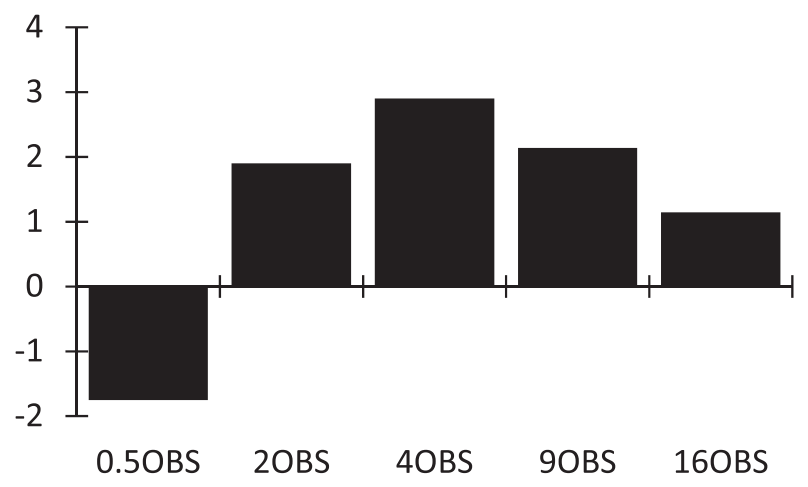

FIG. B1. Normalized forecast RMSE differences of the extra AMSU-A thinning distance tuning experiments without ECM refinement. The normalized forecast RMSEs of the extra experiments, 0.5OBS, 2OBS, 4OBS, 9OBS, and 16OBS are shown for two-day forecasts of $500 \mathrm{hP}$ temperature in global average. The vertical axis denotes the normalized forecast RMSE difference (\%) against CNTL-E, and the horizontal axis denotes experimental names.

\section{APPENDIX B}

\section{Extra Experiments for High Density AMSU-A Assimilation without ECM Refinement}

For comparison with the thinning distance refinement based on the estimated correlation distance shown in the main text, here, thinning distance refinement based on the trial and error approach without the ECM refinement is described. Extra 6 experiments with different thinning distances for AMSU-A were performed on the nearly same NWP system as that described in section 3 except for based on slightly older NWP system used operationally until 2015. The extra experiments are named 0.5OBS, 2OBS, 4OBS, 9OBS, 16OBS, and CNTL-E with 0.5, 2, 4, 9,16 , and 1 times higher density assimilation of AMSU-A.

Figure $\mathrm{B} 1$ shows the forecast RMSE improvement rates for temperature at $500 \mathrm{hPa}$ in 48 -h forecasts against CNTL-E. We see 4OBS is the best in these experiments, and the best thinning distance would be between the settings 2OBS and 9OBS. This result does not contradict the result of the main text. Although, more and more trial and error with different thinning distance experiments would bring more fine estimation, there are still no theoretical support, since MCDs are not estimated, and the estimated thinning distances depend on the ECM used in the DAS. Therefore, as described in the main text, the refinement of thinning distance and the refinement of ECM should be done simultaneously since thinning distances should be determined by the correlation structure of OECMs.

\section{REFERENCES}

Andersson, E., M. Fisher, R. Munro, and A. McNally, 2000: Diagnosis of background errors for radiances and other observable quantities in a variational data assimilation scheme, and the explanation of a case of poor convergence. Quart. J. Roy. Meteor. Soc., 126, 1455-1472, https://doi.org/ 10.1256/smsqj.56511.

Auligné, T., A. P. McNally, and D. P. Dee, 2007: Adaptive bias correction for satellite data in a numerical weather prediction system. Quart. J. Roy. Meteor. Soc., 133, 631-642, https:// doi.org/10.1002/qj.56.

Bannister, R. N., 2008: A review of forecast error covariance statistics in atmospheric variational data assimilation. II: Modelling the forecast error covariance statistics. Quart. J. Roy. Meteor. Soc., 134, 1971-1996, https://doi.org/10.1002/qj.340.

Bauer, P., R. Buizza, C. Cardinali, and J.-N. Thépaut, 2011: Impact of singular-vector-based satellite data thinning on NWP. Quart. J. Roy. Meteor. Soc., 137, 286-302, https://doi.org/ 10.1002/qj.733.

Bonavita, M., L. Isaksen, and E. Hólm, 2012: On the use of EDA background error variances in the ECMWF 4D-Var. Quart. J. Roy. Meteor. Soc., 138, 1540-1559, https://doi.org/10.1002/ qj.1899.

Bormann, N., and P. Bauer, 2010: Estimates of spatial and interchannel observation-error characteristics for current sounder radiances for numerical weather prediction. I: Methods and application to ATOVS data. Quart. J. Roy. Meteor. Soc., 136, 1036-1050, https://doi.org/10.1002/qj.616.

_ A. Collard, and P. Bauer, 2010: Estimates of spatial and interchannel observation-error characteristics for current sounder radiances for numerical weather prediction. II: Application to AIRS and IASI data. Quart. J. Roy. Meteor. Soc., 136, 1051-1063, https://doi.org/10.1002/qj.615.

_ A. J. Geer, and P. Bauer, 2011: Estimates of observationerror characteristics in clear and cloudy regions for microwave imager radiances from numerical weather prediction. Quart. J. Roy. Meteor. Soc., 137, 2014-2023, https://doi.org/10.1002/ qj.833.

_- A. Fouilloux, and W. Bell, 2013: Evaluation and assimilation of ATMS data in the ECMWF system. J. Geophys. Res. Atmos., 118, 12970-12 980, https://doi.org/10.1002/ 2013JD020325.

_- M. Bonavita, R. Dragani, R. Eresmaa, M. Matricardi, and A. McNally, 2016: Enhancing the impact of IASI observations through an updated observation-error covariance matrix. Quart. J. Roy. Meteor. Soc., 142, 1767-1780, https://doi.org/ 10.1002/qj.2774.

Campbell, W. F., E. A. Satterfield, B. Ruston, and N. L. Baker, 2017: Accounting for correlated observation error in a dualformulation 4D variational data assimilation system. Mon. Wea. Rev., 145, 1019-1032, https://doi.org/10.1175/MWR-D16-0240.1.

Daescu, D. N., 2008: On the sensitivity equations of four-dimensional variational (4D-Var) data assimilation. Mon. Wea. Rev., 136, 3050-3065, https://doi.org/10.1175/2007MWR2382.1.

Dalcher, A., and E. Kalnay, 1987: Error growth and predictability in operational ECMWF forecasts. Tellus, 39A, 474-491, https://doi.org/10.1111/j.1600-0870.1987.tb00322.x.

Dee, D. P., 2004: Variational bias correction of radiance data in the ECMWF system. Proc. ECMWF Workshop on Assimilation of High Spectral Resolution Sounders in NWP, Reading, United Kingdom, ECMWF, 97-112.

Derber, J. C., and W.-S. Wu, 1998: The use of TOVS cloud-cleared radiances in the NCEP SSI analysis system. Mon. Wea. Rev., 126, 2287-2299, https://doi.org/10.1175/1520-0493(1998) 126<2287:TUOTCC $>2.0$.CO;2. 
Desroziers, G., and S. Ivanov, 2001: Diagnosis and adaptive tuning of observation-error parameters in a variational assimilation. Quart. J. Roy. Meteor. Soc., 127, 1433-1452, https://doi.org/ 10.1002/qj.49712757417.

_ L. Berre, B. Chapnik, and P. Poli, 2005: Diagnosis of observation, background and analysis-error statistics in observation space. Quart. J. Roy. Meteor. Soc., 131, 3385-3396, https:// doi.org/10.1256/qj.05.108.

Eresmaa, R., J. Letertre-Danczak, C. Lupu, N. Bormann, and A. P. McNally, 2017: The assimilation of Cross-track Infrared Sounder radiances at ECMWF. Quart. J. Roy. Meteor. Soc., 143, 3177-3188, https://doi.org/10.1002/qj.3171.

Errico, R. M., N. C. Privé, and W. Gu, 2015: Use of an OSSE to evaluate background-error covariances estimated by the NMC method. Quart. J. Roy. Meteor. Soc., 141, 611-618, https://doi.org/10.1002/qj.2384.

Golub, G. H., and C. F. Van Loan, 1996: Matrix Computations. 3rd ed. Johns Hopkins University Press, 728 pp.

Hersbach, H., and D. P. Dee, 2016: ERA5 reanalysis is in production. ECMWF Newsletter, No. 147, ECMWF, Reading, United Kingdom, 7, http://www.ecmwf.int/sites/default/files/ elibrary/2016/16299-newsletter-no147-spring-2016.pdf.

Hollingsworth, A., and P. Lönnberg, 1986: The statistical structure of short-range forecast errors as determined from radiosonde data. Part I: The wind field. Tellus, 38A, 111-136, https:// doi.org/10.1111/j.1600-0870.1986.tb00460.x.

Ishibashi, T., 2010: Optimization of error covariance matrices and estimation of observation data impact in the JMA global 4D-Var system. CAS/JSC WGNE Research Activities in Atmospheric and Oceanic Modelling, No. 40, 1.11-1.12.

__ 2011: Tangent linear approximation based observation data impact estimation in 4D-Var. Quart. J. Roy. Meteor. Soc., 137, 1898-1912, https://doi.org/10.1002/qj.871.

__ 2018: Adjoint-based observation impact estimation with direct verification using forward calculation. Mon. Wea. Rev., 146, 2837-2858, https://doi.org/10.1175/MWR-D-18-0037.1.

JMA, 2007: Outline of the operational numerical weather prediction at the Japan Meteorological Agency. Appendix to WMO Technical Progress Report on the Global Data-processing and Forecasting System (GDPFS) and Numerical Weather Prediction (NWP), Japan Meteorological Agency, Tokyo, Japan, accessed 21 May 2020, http://www.jma.go.jp/jma/jmaeng/jma-center/nwp/outline-nwp/index.htm.

_ 2013: Outline of the operational numerical weather prediction at the Japan Meteorological Agency. Appendix to WMO Technical Progress Report on the Global Data-processing and Forecasting System (GDPFS) and Numerical Weather Prediction (NWP), Japan Meteorological Agency, Tokyo, Japan, accessed 21 May 2020, http://www.jma.go.jp/jma/jmaeng/jma-center/nwp/outline2013-nwp/index.htm.

Kadowaki, T., and K. Yoshimoto, 2012: A new inner model with a higher horizontal resolution (TL319) in JMA's Global 4D-Var data assimilation system. CAS/JSC WGNE Research Activities in Atmospheric and Oceanic Modelling, No. 42, 1.9-1.10.
Kalnay, E., 2003: Atmospheric Modeling, Data Assimilation and Predictability. Cambridge University Press, $341 \mathrm{pp}$.

Liu, Z.-Q., and F. Rabier, 2003: The potential of high-density observations for numerical weather prediction: A study with simulated observations. Quart. J. Roy. Meteor. Soc., 129, 30133035, https://doi.org/10.1256/qj.02.170.

Lorenc, A. C., 1986: Analysis methods for numerical weather prediction. Quart. J. Roy. Meteor. Soc., 112, 1177-1194, https:// doi.org/10.1002/qj.49711247414.

Lorenz, E. N., 1982: Atmospheric predictability experiments with a large numerical model. Tellus, 34, 505-513, https://doi.org/ 10.3402/tellusa.v34i6.10836.

Ménard, R., 2016: Error covariance estimation methods based on analysis residuals: Theoretical foundation and convergence properties derived from simplified observation networks. Quart. J. Roy. Meteor. Soc., 142, 257-273, https://doi.org/ 10.1002/qj.2650.

Parrish, D. F., and J. C. Derber, 1992: The National Meteorological Center's spectral statistical-interpolation analysis system. Mon. Wea. Rev., 120, 1747-1763, https://doi.org/10.1175/ 1520-0493(1992)120<1747:TNMCSS > 2.0.CO;2.

Rabier, F., A. McNally, E. Andersson, P. Courtier, P. Undén, J. Eyre, A. Hollingsworth, and F. Bouttier, 1998: The ECMWF implementation of three-dimensional variational assimilation (3D-Var). II: Structure functions. Quart. J. Roy. Meteor. Soc., 124, 1809-1829, https://doi.org/10.1002/qj.49712455003.

Reynolds, C. A., P. J. Webster, and E. Kalnay, 1994: Random error growth in NMC's global forecasts. Mon. Wea. Rev., 122, 1281-1305, https://doi.org/10.1175/1520-0493(1994)122<1281: REGING $>2.0 . \mathrm{CO} ; 2$.

Stewart, L. M., S. Dance, N. K. Nichols, J. R. Eyre, and J. Cameron, 2014: Estimating interchannel observation-error correlations for IASI radiance data in the Met Office system. Quart. J. Roy. Meteor. Soc., 140, 1236-1244, https://doi.org/10.1002/qj.2211.

Teixeira, J., and T. F. Hogan, 2002: Boundary layer clouds in a global atmospheric model: Simple cloud cover parameterizations. J. Climate, 15, 1261-1276, https://doi.org/10.1175/15200442(2002)015<1261:BLCIAG > 2.0.CO;2.

Waller, J. A., S. L. Dance, and N. K. Nichols, 2016: Theoretical insight into diagnosing observation error correlations using observation-minus-background and observation-minusanalysis statistics. Quart. J. Roy. Meteor. Soc., 142, 418-431, https://doi.org/10.1002/qj.2661.

Weston, P., W. Bell, and J. Eyre, 2014: Accounting for correlated error in the assimilation of high-resolution sounder data. Quart. J. Roy. Meteor. Soc., 140, 2420-2429, https://doi.org/ 10.1002/qj.2306.

Wilks, D. S., 2011: Statistical Methods in the Atmospheric Sciences. 3rd ed. International Geophysics Series, Vol. 100, Academic Press, 704 pp.

Žagar, N., M. Horvat, Ž. Zaplotnik, and L. Magnusson, 2017: Scale-dependent estimates of the growth of forecast uncertainties in a global prediction system. Tellus, 69A, 1287492, https://doi.org/10.1080/16000870.2017.1287492. 